\title{
Einleitung.
}

\section{Die Handschriften.}

Unsere Überlieferung der armenischen Version der Chronik des Eusebius beruht auf zwei Codices:

1) einem Jerusalemer Codex, den wir mit $G$ bezeichnen, welcher vermutlich aus der Zeit von 1294-1307 n. Chr. stammt ${ }^{1}$ und Ausgangs des 18. Jhd.s aus der Bibliothek des armenischen Patriarchates von Jerusalem an die Bibliothek des armenischen Patriarchalseminars von Konstantinopel überging ${ }^{2}$;

2) einem Etschmiadziner Codex, den wir mit $\mathrm{E}$ bezeichnen, welcher in der Etschmiadziner Metropolitan-Bibliothek aufbewahrt wird, gleichwie G der Zeit des 13.-14. Jhd.s entstammen mag und i. J. 1898 auf Anregung der Kgl. Berliner Akademie der Wissenschaften photographisch reproduciert und ediert worden ist ${ }^{3}$.

Beide Codices stimmen in der äußeren Anlage Seite für Seite wesentlich überein, die einzelnen Seiten in Rundminuskel von $\mathrm{E}$ sowohl als $\mathrm{G}$ bestehen regelmäßig aus 36 (od. 35) Zeilen. Die vereinzelten Ab-

1) Nach Petermann (Ed. Schoene II p. XLIX).

2) Zur näheren Beschreibung des Cod. G vgl. Aucher Ed. Intr., Schoene Ed. II p. XLIX sq.

3) Die äußere Anlage und Ausstattung von $\mathbf{E}$ stimmt wesentlich mit $\mathrm{G}$ überein, abgesehen von den in $\mathrm{G}$ vorhandenen Schreibernotizen (Postscripta). Inwiefern die von dem Etschmiadziner Bibliothekskatalog (Tifl. 1865) p. 187 Nr. 1684 gemachte Datierung dieses Codex, wonach derselbe aus d. J. 1695 n. Chr. stammen würde, zutrifft, kann hier bei dem Fehlen jeder Spur eines derartigen Datums in der phothographischen Reproduction des Codex nicht entschieden werden. Unzutreffend ist jedenfalls die in demselben Katalag gegebene Notiz: ser (Cod. E) hat keine Gedenkschrift, bloß einen Index . Vom Index ist in der photogr. Ausgabe keine Spur vorhanden. 
weichungen bezüglich der Stellung der Lemmata zu den betreffenden Jahren der fila regnorum im Kanon sind auf nachlässige Copistenarbeit oder Textzerrüttung zurückzuführen. Beide Codices zeigen gemeinsam eine Anzahl größerer Defecte oder Textlücken. Durch Blätterausfall bzw. Verstümmelung oder Copistenüberschlagung fehlen sowohl in $\mathrm{E}$ als in $\mathrm{G}$ :

a) der Anfang der zwischen dem I. Buch und dem Kanon eingeschobenen Königslisten, und zwar die ganze Serie der Assyrerkönige und die Hebräerfürsten bis zu Jephtai;

b) der Schluß der Königslisten bzw. des Todeskataloges der römischen Kaiser von Gordian bis zu Konstantin d. Gr.;

c) der Prolog zum Kanon nebst dem Anfang des Kanons bis zum J. Abrah. 344, durch Ausfall von ungefähr 5 Blättern;

d) zwei Seiten Text, durch Ausfall eines Blattes, inmitten des Kanons, umfassend ein Intervall von 70 Jahren, vom J. Abr.1030 bis z. J. Abr. 1100;

e) zwei Seiten Text, durch Blattausfall, umfassend einen Zeitraum von 55 Jahren v. J. Abr. 1166 bis z. J. Abr. 1221;

f) wohl auch die letzte Seite des Kanons, durch Ausfall des letzten Codexblattes, umfassend 26 Jahre, v. J. 16 Diokletians bis zu den Vicennalien Konstantins, oder v. J. Abr. 2320-2343 ${ }^{1}$.

Scheinbar liegt auch ein größerer Textdefect am Ende des I. Buches vor, wo die in Aussicht gestellte Chronographie der römischen Kaiserzeit nebst der einschlägigen Consuln-Liste vermißt wird. Die Frage, ob hier wirklich Textverstümmelung anzunehmen sei, soll im folgenden (p. XXVIII ss.) noch eingehender geprüft werden.

Aber auch kleinere Lücken und Defecte sind beiden Codices gemeinsam; so z. B. Chron. I., Abschnitt Römergeschichte, in dem Excerpt aus Dion. Halikarn. der Satz: „Romilos nämlich, der Gründer der Stadt, heißt es, habe 37 Jahre die Gewalt innegehabtヶ; ibid. im Verzeichnis der Römerkönige die Namen Amulios, Silvios und Avẹntios; ferner im Kanon die gemeinsamen Defecte zu J. Abr. 1867, wo das Datum ,668 Jahres und zu J. Abr. 2296, wo der Bischofsname sEutichianoss ausgefallen ist u. a. m. Übereinstimmung zwischen beiden Codices zeigt sich weiter auch in einer langen Serie von Copistenversehen, Verwechslungen und graphischen Irrtümern, auf die bereits von Petermann (Ed. Schoe. II p. LI sqq und I p. IX $\mathrm{sqq}^{2}$ ) hingewiesen ist, und

1) Vgl. indessen hiergegen das im folgenden S. XXX-XXXI Bemerkte.

2) Die dort von $\mathrm{P}$. angestellte Vergleichung zwischen Codd. $\mathrm{G}$ und $\mathrm{N}$ gilt, worauf hier ausdrücklich hingewiesen sei, ohne daß $P$. es ahnte, auch wesentlich für das Verhältnis von $\mathrm{G}: \mathrm{E}$. Denn $\mathrm{N}$ ist nichts weiter als eine (verschlechterte) Copie von E. 
die im Laufe unserer Textwiedergabe ausführlich zur Darstellung kommen werden. Namentlich lehrreich sind solche gemeinsame graphische Fehler beider Codices, die aus Verlesung bzw. Verwechslung von Majuskeln hervorgegangen sind: Aseres st. Ameres, Sarakos st. Tarakos, Sente st. Menthe Armes st. Arses, Miasun st. Miamun; ferner êm st. êt, sirẹr st. tirẹr und umgekehrt, etc., lauter Versehen, die auf Verwechslung von $\mathbf{D}-\mathbf{W}$, $\mathbf{S}-\mathbf{l}, \mathbf{l}-\mathbf{S}$ beruhen. Hieraus folgt zugleich, daß beide Codices aus einer in Majuskelschrift verfaßten gemeinsamen Urquelle hervorgegangen sind.

Daß nun nicht etwa der eine der beiden Codices direct vom anderen abhängig und aus ihm geflossen sein kann, ergibt sich aus den immerhin trotz jener Berührungspunkte zwischen ihnen bestehen bleibenden Abweichungen.

Cod. E weicht von $\mathrm{G}$ ab durch Verschiedenheit der Lesart einzelner Wörter und Satzteile, sowohl in graphischer als in grammatischer Hinsicht; so zwar, daß im allgemeinen die Lesung nach $\mathrm{E}$ die vorzüglichere ist (vgl. die diesbezüglichen Belege Ed. Schoe. II p. LII) ${ }^{1}$; sodann auch durch vollständigere Form des Textes. Denn G zeigt zahlreiche Defecte, die sich aus $\mathrm{E}$ ergänzen. So fehlen z. B. nach $\mathrm{G}$ im Kanon die Lemmata ad a. Abr. 981, a. Abr. 1259 , a. Abr. 1283, a. Abr. 1746, a. Abr. 2135, a. Abr. 2181 u. a.m., welche uns sämtlich im Cod.E erhalten sind. Auch sind manche mehr oder weniger bedeutende Textlücken, die $G$ in T. I aufweist, in E nicht vorhanden.

Hieraus ergibt sich: beide Handschriften $\mathrm{G}$ und $\mathrm{E}$ sind unabhängig von einander aus einem gemeinsamen, in Majuskel verfaßten, Archetypus geflossen, welchen $\mathrm{G}$ in mehr corrupter und defecter Fassung wiedergibt, während $\mathrm{E}$ den besseren, vollständigeren Urtext bewahrt hat.

Lediglich als jüngere, verschlechterte, teilweise ganz verwahrloste Abschrift aus $\mathrm{E}$ stellt sich Ms. $\mathrm{N}$ dar ${ }^{2}$, eine im Jahre $1696 \mathrm{n}$. Chr. in Tokat verfertigte, für die Textherstellung der Chronik ziemlich belanglose Arbeit ${ }^{3}$.

1) Das dort von $P$. über $N$ Gesagte gilt in erster Linie von $\mathbf{E}$.

2) Dieser unselbständige Charakter von $\mathrm{N}$ als eines Apographs von $\mathrm{E}$ ist von Petermann völlig verkannt worden, was indessen leicht begreiflich wird, da P. nur eine mangelhafte fragmentarische Collation aus $\mathbf{E}$ zur Verfügung stand. Doch erkannte aus einer gegenseitigen Vergleichung schon $P$. die engere verwandt. schaftliche Verbindung zwischen $\mathrm{N}$ und $\mathrm{E}$.

3) Der Codex wurde von dem Mechitharisten P. Nerses um 1856 für die Venezianische Mechitharistenbibliothek erworben, wo er von Petermann eingehend collationiert wurde. 


\section{Die älteren Ausgaben.}

Die erste und bisher einzige Originalausgabe, die von Aucher im Jahre 1818 auf Grund einer eigens hierzu aus dem Konstantinopler Codex hergestellten Copie ${ }^{1}$ veröffentlicht wurde, konnte schon deshalb keine streng kritische werden, weil sie den besseren, für eine Ausgabe in erster Linie in Betracht kommenden Codex $\mathrm{E}$ nicht berücksichtigte. Infolgedessen leidet das Auchersche Werk, trotz der darin entfalteten universellen Gelehrsamkeit und trotz eines sich turmhoch aufstapelnden wissenschaftlichen Apparates, an empfindlichen Schwächen und Mängeln auch in der Textinterpretation, zumal überdies Aucher mit den ihm damals erst beschränkt zu Gebote stehenden philologischen Hilfsmitteln nicht immer richtig den Sinn des schwierigen armenischen Textes zu erfassen vermochte, derart, daß seine Übersetzung sich von dem Armenisten Saint-Martin und nach diesem von Niebuhr den Vorwurf der Oberflächlichkeit und Unzuverlässigkeit gefallen lassen mußte ${ }^{2}$.

Mit Aucher rivalisierend und ihm zuvorkommend hatte schon kurz vorher in demselben Jahre 1818 dessen Ordensgenosse, der Philolog Zohrab in Verbindung mit A. Mai, der die italienische Übersetzung des Armeniers ins Lateinische übertrug, eine lateinische Ausgabe der Chronik erscheinen lassen ${ }^{3}$. Wie der Auchersche so fußt auch der Zohrabsche Text auf Cod. G, der jedoch in einer minderwertigen, der

1) Aus Cod. G, der 1787 aus Jerusalem an die armenische Kolonie von Kpol gekommen war, nahm 1790 Georg Johannesian für Aucher eine Abschrift, sodann 1793 eine zweite, genauere. Dieser Apographus v. J. 1793, der später von Aucher in $\mathrm{K}$ pol mit dem Originalcodex verglichen und controlliert wurde, bildet die Grundlage der Aucherschen Ausgabe.

2) Vgl. betr. die St.-Martinschen Angriffe „Journal des Savans a févr. 1820 p. 97-112; betr. die Niebuhrschen vgl. dessen Aufsatz »Historischer Gewinn aus der armenischen Ubersetzung der Chronik des Eusebius 1819 (Abhdlg. d. hist.phil. Cl. d. Kgl.Pr.Akad.d. Wiss.1820-21, Berl. 1822; Kl. Schr. Niebuhrs 1 179-304).

3) Wiewohl Aucher bereits 1795 seine Editio der Chronik druckfertig vorliegen hatte, so zögerte er doch, offenbar in der Hoffnung, weitere hdschr. Quellen zu deren größerer Sicherstellung erlangen zu können, volle 23 Jahre mit der Verrffentlichung. Inzwischen hatte der Mechitharist Zohrab, derselbe der i. J. 1793 die zweite Abschrift von Cod. $\mathrm{G}$ aus $\mathrm{Kpol}$ nach Venedig gebracht hatte, heimlich und ohne Erlaubnis seiner Ordensbehörde die erste Copie desselben Codex nach Mailand ausgeführt und dort im Jahre 1818 die erste Eusebiusausgabe mit A. Mai in lateinischer Fassung publiciert. Aucher, der nun auch schleunigst seine Ausgabe nachfolgen ließ, geriet unverdienterweise in den Verdacht des Plagiats; es entstand jene leidige, peinliche Polemik, worüber zu vgl. Ed. Schoe. II p. XLVI sqq. 
Aucherschen nicht ebenbürtigen Copie $^{1}$ benutzt ist; ein Nachteil, der auch dadurch kaum aufgewogen wird, daß die Zohrabsche Textvorlage im ersten Teile der Chronik die Varianten einer anderen Handschrift, die wohl nur $\mathrm{N}$ sein kann, collationiert, übrigens in meist corrupter Lesart. Was sodann die Interpretation betrifft, so verfährt im Gegensatze zu der Aucherschen Übersetzung, die sich wenigstens getreu an das Original hält, diese äußerst willkürlich. Sie stellt sich offenbar nicht sowohl dar als eine adäquate Wiedergabe des armenischen Originals, denn vielmehr als einen Versuch der Reconstruction der ursprünglichen Eusebius-Chronik an der Hand des armenischen Textes in Verbindung mit den anderweit erhaltenen Bruchstücken desselben Eusebius-Werkes. Zohrab-Mai ist bestrebt, nicht sowohl den eigentlichen Sinn des Armeniers aus seiner Vorlage herauszulesen, als vielmehr den des Original-Eusebius hineinzuinterpretieren. So wird denn, namentlich bei schwierigen Stellen und Fällen von Abweichung der armenischen Version von den uns erhaltenen entsprechenden griechischen Textfragmenten, regelmäßig unter Preisgabe der armenischen Lesung die entsprechende griechische recipiert. Infolge dieser Interpretationsmethode gestaltet sich diese vermeintliche lateinische Editio ZohrabMai der Eusebius-Chronik, wiewohl vor der Aucherschen den Vorzug größerer Lesbarkeit und tieferen Eindringens in das Textverständnis zur Schau tragend, zu einer unvergleichlich weniger kritisch zuverlässigen als jene.

So war es denn ganz naturgemäß, daß der Orientalist H. Petermann, dem i. J. 1866 die Aufgabe zufiel, anstelle jener zwei veralteten lateinischen Versionen eine neue, kritisch zuverlässige für die 1866-1875 erscheinende Schoenesche Eusebiusedition ${ }^{2}$ zu schaffen, sich, in bewußtem Gegensatze zu Zohrab-Mai, in seiner Publication der armenischen Eusebius-Chronik in lateinischer Übertragung an Aucher anlehnte. Bezeichnete somit die Petermannsche Lateinausgabe eine in gewissem Sinne gerechtfertigte Reaction gegen Zohrab-Mai und deren Anhänger und eine gerechtere Würdigung der hohen philologisehen Verdienste

1) Vgl. die vorige Note.

2) Eusebi Chronicorum libri duo ed. Alfred Schoene.

Vol. II. Chronicorum Canonum quae supersunt . . . A Armeniam Versionem latine factam e libris manuscriptis recensuit H. Petermann. Hieronymi Versionem e libris manuscriptis recensuit A. Schoene. Syriam epitomen latine factam e libro Londinensi recensuit Roediger. Berolini 1866.

Vol. I. Chronicorum liber prior. Liber I. Armeniaun Versionem latine factam ad libros manuscriptos recensuit $H$. Petermann. Graeca fragmenta collegit et recognovit, Appendices chronographicas sex adiecit A. Schoene. Berolini 1875. 
eines Aucher, den er gegen Niebuhrs und Saint-Martins teils unverständige, teils böswillige Kritik in Schutz nahm ${ }^{1}$, so war es gleichwohl für diese neue lateinische Version ein höchst verhängnisvoller Mißgriff, daß Petermann in allzu pietätvoller, zuversichtlicher Anlehnung an die Auchersche Autorität dessen Textinterpretation als Norm zugrunde legt und nur zu häufig ohne weitere Nachprüfung mit den Aucherschen Fehlern und Mängeln übernimmt. Dies gilt besonders für den I. Teil der Chronik, wohingegen im Kanon sich der Herausgeber selbständiger zeigt. Zwar hat die Petermannsche Version der Chronik vor ihren zwei Vorgängerinnen den entschiedenen Vorzug voraus, daß hier zum ersten Male die bessere Textform des Cod. E, wenn nicht durchweg im authentischen Original, so doch wenigstens in dessen Repräsentanten, Ms. N, herangezogen und verwertet ist ${ }^{2}$. Gleichwohl bleibt auch ihr der Charakter allzu conservativer und seichter Kritik als schwerer Schaden anhaften.

\section{Die vorliegende Ausgabe, ihre Einrichtung und Text- herstellung mit Bezug auf Anlage und Ausstattung der handschrift- lichen Chronik.}

Nach dem vorhin Gesagten muß es als höchst zw eifelhaft erscheinen, ob überhaupt für derartige Übertragungen, wie die der Eusebius-Chronik, die lateinische Sprache das richtige Mittel ist. Vermag sie doch auch nicht annähernd die Feinheiten und den formalen und syntaktischen Reichtum der armenischen, die sich in wunderbarer Schmiegsamkeit und Biegsamkeit dem Originale fügt und anbequemt, zu erreichen und wiederzugeben. Das Fehlen eines Artikels ist in ihr insbesondere ein Hemmnis

1) Vgl. im einzelnen die diesbezüglichen Ausführungen Petermanns in Ed. Schoe. II p. XLVI sqq.

2) Für den II. Teil, den Kanon, war P. ausschließlich auf Codd. GN angewiesen gewesen. Für den I. Teil, die 1875 erscheinende, eigentliche Chronographie, kam als weiteres Hilfsmittel hinzu eine ihm auf Mommsens Betreiben und durch Verwendung des Chrimer Erzbischofs Aiwazowski aus dem Etschmiadziner Codex hergestellte Collation, die indessen sich auf die Stücke I S. 386-397 und II S. 19-20 nach Ed. A beschränkte, also eine sehr mangelhafte war. Nicht glücklicher waren P.s Bemühungen um eine wissenschaftliche Collation des Originalcodex G: eine zu diesem Zwecke eigens von $\mathrm{P}$. nach Kpol unternommene Reise blieb erfolglos; confessionelle Eifersucht hintertrieb eine liberale Einsichtgabe in den Codex (vgl. Ed. Schoe. II p. XLVIII). 
Einleitung. IIl. Einrichtung u. Textherstellung der vorliegenden Ausgabe XVII

zur genauen Wiedergabe der fremden Eigennamen. So zeigt uns denn auch naturgemäß das Petermannsche, in diesem Werke angewandte System der Transcription das wunderliche Schauspiel der Beibehaltung des barbarischen Wortstammes mit Latinisierung der Endung bzw. Flectierung mittels lateinischer Casussuffixe; auf solche doppelten Barbarismen, wie Ptlomaeus für arm. Ptlomeos, Triptlomus für Triptlomos, Peleponesus für Peleponesos, Kroisus od. Krisus für Kroisos, Krisos etc. hat schon A. v. Gutschmid rügend hingewiesen ${ }^{1}$; ganz abgesehen von anderen, wohl gleichfalls der lateinischen Sprache zu Gefallen zugelassenen Mängeln der Transcription, wie z. B. die Nichtunterscheidung der Quantität langer und kurzer Vokale u. a. m.

Näherliegend wäre entschieden eine Rückübersetzung in das Griechische. So hat denn auch u. a. Gutschmid (Kl. Schr. 1, 441) sein Bedauern dahin geäußert, daß dies für die Petermannsche Version nicht geschehen, da doch das Armenische sich sauf das Engste an das Griechische anschmiegt, so daß eine Rückübersetzung in der Weise der Lagardeschen die gegebene Form gewesen wäre, dạ Werk klassisch gebildeten Lesern näher zu bringen «.

Allein Gutschmid geht hier von einer unerwiesenen Petitio principii aus, indem er ohne weiteres annimmt, unsere armenische Version sei aus einer griechischen Vorlage geflossen; während hingegen der Orientalist Petermann z. B. eine syrische Quellenvorlage annimmt ${ }^{2}$. Mögen auch teilweise Gräcismen vorliegen und die Form der Eigennamen vorwiegend die griechische sein, so folgt hieraus noch keineswegs, daß Arm. durchgehends und ausschließlich aus griechischer Vorlage geflossen sei. Ist dem aber so und bleibt die Möglichkeit, daß ein syrisches Original wenigstens mit als Quellengrundlage benutzt worden sei, so wäre es verkehrt, den also durch syrisches Mittel hindurchgegangenen Armenier durchgehends gräcisieren zu wollen, geschweige denn gar das also durch vermeintliche sRückübersetzung gewonnene Product für den wiedererstandenen griechischen OriginalEusebius $\mathrm{zu}$ halten. Es wäre dies eine allzu gewagte und der subjectiven Willkür einen zu breiten Spielraum lassende Methode.

Unter diesem Gesichtspunkte und von derselben Ansicht ausgehend hat die Kirchenvätercommission der Kgl. Preuß. Akademie der Wissenschaften im Frühjahr 1906 den Beschluß gefaßt, die Eusebius-Chronik so sicher als möglich in deutscher Übersetzung herauszugeben, als

1) A.v. Gutschmid, Uber Schoenes Ausgabe der Chronik des Eusebius (Kl. Schr. I S. $441 \mathrm{f})$.

2) Ed. Schoe. II p. LIII. 
Vorarbeit zu einer künftigen Edition des Hieronymus-Kanon und sodann des Original-Eusebius, und uns mit dieser Aufgabe betraut.

Diese deutsche Version, die im Folgenden gegeben wird, ist nach zwei Grundsätzen ausgearbeitet: sie soll werden eine formell und eine inhaltlich adäquate.

1) Formell adäquat, d. h. eine wörtliche, auch möglichst die Satzconstruction und das sprachliche Colorit des Armeniers widerspiegelnde. Dazu gehört insbesondere die genaue Wiedergabe a) der Eigennamen, b) der graphischen Anlage.

a) Eigennamen werden transcribiert in der Form, in der sie beim Armenier vorkommen, also unter Belassung nicht bloß der Stammlautform, sondern auch der Endung. Von diesem Princip wird abgewichen nur in Fällen, wo die Transcriptionsform von der uns geläufigen allzusehr verschieden ist und Undeutlichkeiten veranlassen könnte, so z. B. arm. hrea sJude», hreastan "Judäa«, Asori sSyrer», Asorikh "Syrien neben Asorestan "Assyrien«, Asorestanea sAssyrerc, Parsik „Perser Mar sMeder .

Etwaige Inconsequenzen in der Anwendung dieses Systems dürften teils in dem Bestreben der Bewahrung des Originalcolorits, teils hinwieder in demjenigen nach Lesbarkeit ihre Entschuldigung finden. Derselbe Gesichtspunkt der größeren Lesbarkeit war denn auch ausschlaggebend und bestimmend für die Methode des Ausdrucks der Laute des armenischen Alphabets. Nicht darum konnte es sich handeln, eine streng phonetisch adäquate Transcription der Originallaute zu geben, sondern vielmehr die uns geläufigere Entsprechung des transcribierten Originalworts mit Abstreifung der speziell armenischen Lautnüance zu erzielen. Daher umschreiben wir arm. $\ldots$ nicht mit Petermann durch kh sondern durch ch, also eprumnu nicht etwa khristos sondern christos, eppunqninu nicht etwa khrisogonos sondern chrisogonos; analog setzen wir die armenischen Lautgruppen $\boldsymbol{e}^{\boldsymbol{u}}, \boldsymbol{\psi} \boldsymbol{u}$ nicht wie Petermann in ihr streng linguistisch entsprechendes Lautbild khs, phs um, sondern in die uns geläufigen $\mathbf{x}$ resp. ps $(\psi)$ :

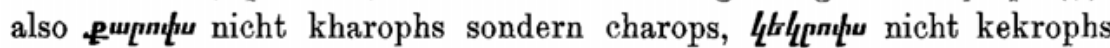
sondern kekrops, pulsmpuilsu nicht khsenophanes sondern xenophanes etc. Höchst ungeeignet und störend und den wirklichen lautlichen Sachverhalt trübend schien uns aus analogem Grunde auch die pedantische Transcription von originalem $\boldsymbol{m} \mathbf{L}, \boldsymbol{t} \boldsymbol{L}, \boldsymbol{k} \boldsymbol{k}+$ Vocal, $\boldsymbol{u} \boldsymbol{J}, \boldsymbol{y}$ + Vocal durch av, ev, iv + Vocal, ay, oy + Vocal, wo doch in der Originalsprache einfacher Diphthong entspricht; es soll daher im folgenden ein 4 ujisınnu zu Kointos, nicht mit Petermann zu Kojintos werden, ein uJtrnųuи zu Aeropas, nicht zu ajeropas etc. So geben wir 
Einleitung. III. Einrichtung u. Textherstellung der vorliegenden Ausgabe XIX

denn auch das arm. nL der Eigennamen nicht durch seine graphische Entsprechung ov, sondern durch seinen phonetischen Wert ô wieder. Folgerichtig müßte auch arm. scharfes $\boldsymbol{\nu}$ als Entsprechnng von griech. $\sigma \sigma$ durch ss sowie sonstige Vereinfachung, von originaler Doppelconsonanz in unserer Transcription ausgeglichen werden. Zum Teil ist dies geschehen, wiewohl nicht in streng consequenter Durchführung. Umgekehrt ist unbedenklich arm. $\mathbf{Z}$ gleichwie $L$ durch das gemeinsame 1 wiedergegeben, auch $\boldsymbol{n}$ nur in zwingenden Fällen von $\boldsymbol{r}$ geschieden worden. Noch eigens ist hervorzuheben, daß das Zeichen e als Transcription von $\boldsymbol{\xi}$, wiewohl lautgeschichtlich im allgemeinen der e-Laut aus ey hervorgegangen ist, nicht quantitativ, sondern qualitativ von $\boldsymbol{\theta} \boldsymbol{t}$ verschieden ist, indem es ein offenes, nach $\ddot{a}$ hinneigendes E darstellt, während $\boldsymbol{t}$ (e) dem geschlossenen, hellen, an i anklingenden und z. T. auch mit diesem wechselnden E-Laut entspricht; daher denn

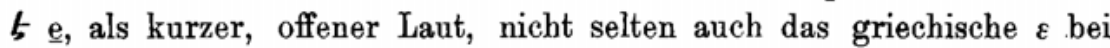
Wiedergabe griechischer Eigennamen ersetzt.

b) Die graphische Anlage soll soweit als möglich in ihrer ursprünglichen Überlieferungsform gewahrt bleiben, so daß im Kanon vor allem die einzelnen Seiten den betreffenden des Originalmanuscripts entsprechen. Und da bei einem synchronistischen Werke wie der Eusebische Kanon die äußere Ausstattung und lineare Gliederung keineswegs accessorisches Beiwerk, sondern vielmehr wesentlicher, erst das richtige Verständnis des Textes ermöglichender und den Sinn bestimmender Bestandteil ist, und es demnach vor allem darauf ankommt, die ursprüngliche Form des Kanons soweit als möglich zu eruieren und festzustellen, so dürfte es angezeigt sein, in dieser Beziehung auf einige Punkte der äußeren Ausstattung in der Überlieferung des Armeniers behufs näherer Prüfung hier einzugehen.

Rand-Titel. - Außer größeren, generellen Titeln und Überschriften, durch die des Werkes Einteilung bezeichnet ist, sind noch Seitentitel am unteren Codex-Rande überliefert, so zwar, daß regelmäßig von zwei einander en regard geschriebenen Codexseiten nur die rechte Seite den den Inhalt summarisch bezeichnenden Fußtitel erhält. Diese marginalen Fußtitel sind der Reihe nach bei Cod. $\mathrm{E}$ folgende:

I. Chronographie.

Cod. E pp. 4, 6, 8, 10, 12, 14, 16, 18 (= Ed. p. 4-25): ChaldäerGeschichte.

" pp. 20, 22, 23 (= Ed. p. 25-32): Assyrer-Geschichte. 
Cod. E pp. [24 $\left.{ }^{1}\right], 26,28,30,32,34,36,38,40,42,44,45$ und 46 (= Ed. p. 34-62): Ebräer-Chronographie ${ }^{2}$.

$" \quad$ pp. 47, 52, 53, 54 (= Ed. p. 62-64, 70-74): EgypterChronographie.

pp. 48, 49, 50, 51 (= Ed. 65-69): Egypter-Dynastie(en).

” pp. 56, 57, 58, 59, 60 (= Ed. 74-80): Egypter-Ptlomäer (bzw. p. 58-59: Egypter - Ptlomäer - Kleopatra).

p. 61 (= Ed. p. 80-81): Griechen-Chronographie. Sikyon.

p. 62 (= Ed. p. 83-85): Griechen-Argiver.

p. $63,64,65$ (Ed. p. 85-89): Griechen-Athener.

p. $66-73$ (= Ed. p. 89-103): Olympiaden.

p. 74 (= Ed. p. 104 f): Korinther-Könige.

p. $75-76$ (=- Ed. p. 105-107): Lakedämonier-〈Könige〉.

p. $76-81(=$ Ed. p. 107-114): Makedonier-Könige.

p. $81-82$ (= Ed. p. 114-116): Thettaler-Könige nach Alexander.

p. 83-89 (= Ed. p. 117-124): Asianer und Syrer-Könige nach Alexander ${ }^{3}$.

p. 90,92 und 93 (= Ed. p. 125 ff): Römer-Geschichte.

p. 91 (=Ed. p. 126-128): Wer die Römer seien und woher.

p. 94-97 (Ed. p. 129-136): Von der Gründung (Erbauung) der Römer-Stadt.

p. 98-99 (Ed. p. 136-140): Der Römer Urzeiten-Könige ${ }^{4}$.

p. 100-101 (Ed. p. 140-143): Römer-Könige.

p. $102^{\mathrm{I}}$ (= Ed. p. 144): Könige Judas.

p. $102^{\mathrm{II}}(=$ Ed. p. 145): Könige Israels.

p. $103^{I}(=$ Ed. p. 145-46): Könige Babelons. SikyonierKönige.

p. $103^{\mathrm{II}}, 104$ (= Ed. p. 146-148): Egypter-Könige.

p. $105^{\mathrm{I}}(=$ Ed. p. 148): Argiver-Könige.

p. $105^{\mathrm{II}}(=$ Ed. p. 148-49): Athener-Könige. Fürsten.

p. 106 (= Ed. p. $149 \mathrm{ff}$ ): Titel fehlt.

p. $107 \mathrm{I}$ (=Ed. p. 150-51): Makedonier-Könige.

p. 107 II $(=$ Ed. p. 151): Lyder-Könige.

p. $108^{I}$ (= Ed. p. 152): Perser-Könige.

1) Da diese Codexseite noch ausschließlich durch die Königslisten der Meder, Lyder und Perser ausgefüllt ist, so ist der Titel "Ebräer-Chronologie" um eine Seite zu früh angesetzt.

2) Teilweise auch "Hebräer-Chronographien*.

3) Pg. 87: „Syrer und Asianer Könige nach Alex.«.

4) Pg. 99 Var.: sDer Römer Urkönige (erste Könige) 
Einleitung. III. Einrichtung u. Textherstellung der vorliegenden Ausgabe XXI

Cod. E p. $108^{\mathrm{II}}$ (= Ed. p. 152-53): Ptlomäer-Egypter-Könige.

p $1^{109^{\mathrm{I}}}(=$ Ed. p. 153-54): Makabäer.

" p. $109^{\mathrm{II}}(=$ Ed. p. 154-55): Tod der Kaiser.

II. Kanon.

Cod. E p. 110 , 112,114 (= Ed. p. $156,158,160)$ : Knechtschaft Israels in Egiptos.

p. 116 (= Ed. p. 162): ohne Titel.

" p. 118 (= Ed. p. 164): Godoniel.

p. 120 (= Ed. p. 166): Aôd.

" p. 122 (= Ed. p. 168): Gedeon.

" p. 124 (= Ed. p. 170): Jair.

" p. 126 (= Ed. p. 172): Heli der Priester.

" p. 128 (= Ed. p. 174): Samuel.

p. 130 (Ed. p. 176): Solomon.

" p. 132, 134 (= Ed. p. 178, 180): ohne Titel.

" p. 136 (= Ed. p. 182): Ezekkia.

" p. 138 (= Ed. p. 184): Manase.

" p. 140 (= Ed. p. 186): Sedekia.

" p. 142 (= Ed. p. 188): Kyros.

" p. 144 (= Ed. p. 190): Zorababel.

" p. 146 (= Ed. p. 192): Titel fehlt.

" p. 148 (= Ed. p. 194): Neemi der Ebräer. Dareh der Kebsensohn.

" p. 150 (=Ed. p. 196): Der Makedonier Philipos.

Die folgenden Seiten bis zum Schluß des Kanons sind ohne Fußtitel. Auch hierin verrät sich wieder die mehr rudimentäre äußere Ausstattung des Schlußteils des Kanons beim Armenier. Denn daß diese Titelausstattung ursprünglich eine durchgreifende, über das ganze Werk sich erstreckende war, kann nicht bezweifelt werden. Namentlich für den Kanon erscheinen diese charakteristischen Marginal-Stichtitel als treffliches Hilfsmittel sowohl zur Orientierung innerhalb des Ganzen als zur kurzen und bündigen Kennzeichnung der einzelnen Kanon-Tafeln. Daher sie nicht nur als echter Bestandteil der armenischen Version, sondern auch als ursprünglich eusebianisch zu gelten haben werden.

Der historische Text des Kanons und seine Stellung zu dem Zahlensystem. - Der historische Text des Kanons ist auf zwei Columnen verteilt, die, je am äußeren und inneren Seitenrand, außerhalb des Zahlengerüstes der fila regnorum stehen. Wie sich aus einer Be- 
trachtung der einzelnen Kanontafeln ergibt, ist durchschnittlich die überwiegende Masse der Lemmata auf die rechte Randcolumne vereinigt. Die Mehrzahl der Lemmata betr. die Profangeschichte finden sich auf dem rechten Rande. Dagegen ist die linke Randspalte ursprünglich und eigentlich den Lemmata der Biblischen Geschichte reserviert gewesen. Diese ursprüngliche Anordnung, die dem Prototyp des Armeniers eigen war, tritt, wiewohl verblaßt und entstellt, doch noch unverkennbar in unsern Codices hervor. In diesen erweist sich nämlich da, wo die linke Textcolumne Lemmata aus der profanen Geschichte enthält, diese Stellung so gut wie immer lediglich als eine secundäre, indem zur Entlastung des rechten Randes regelmäßig in solchen Fällen, wo durch Zusammentreffen mehrerer Lemmata auf ein und dasselbe Regentenjahr, der rechte Seitenraum nicht ausreichte, der überschüssige Profantext, soweit aus räumlichen Rücksichten nötig, auf den linken Rand übertragen wurde. Seltener und mehr willkürlich erscheinen umgekehrt Lemmata der Hebräer- oder Bibl. Geschichte nach rechts versetzt, so z. B. p. 178. Mehreres dürfte auf willkürlicher Copistenänderung beruhen, so z. B. p. 156 die Stellung des untersten, profanen Lemmas am linken Seitenrand, wofür hier, bei genügendem Raum am rechten Rand, kein eigentlicher innerer Grund vorlag. Arm. stimmt danach weder mit der Zwei-Columnen-Sippe noch mit der Sippe des Spatium Historicum der Hieronymus-Chronik überein; denn dort stehen die zwei Textcolumnen innerhalb der fila regnorum und hier, beim Spatium Historicum, sind sowohl profane als biblische Lemmata in eine einzige Textcolumne vereinigt. Erwägt man nun, daß die Recension des sog. Spatium Historicum, die auch nach Schoenes Zugeständnis auf einer sehr alten, bestgesicherten Überlieferung ruht und dem Texte nach der anderen, der Zwei-Columnen-Sippe an Ursprünglichkeit und Güte überlegen ist, übereinstimmend mit dem Armenier und abweichend von der Sippe der zwei inneren Textcolumnen, das filum regni Hebraeorum an erster Stelle, und zwar vor dem des Assyrer-Reiches, in der Zahlentabelle überliefert, und daß diese dominierende Stellung der Hebräer ganz dem Geiste der Eusebischen Einleitung sowie der nach kirchengeschichtlichen Hauptgesichtspunkten gestalteten Anlage und Disposition des Gesamtwerkes und des Kanons insbesondere passend entspricht, so wird wahrscheinlich diese äußere Anordnung als die ursprünglichere zu gelten haben und dürfen wir annehmen, daß nicht bloß dem Texte nach (wie es Schoene a. a. 0. dargetan hat), sondern auch in der äußeren, tabellarischen Anlage und in der Anordnung der Lemmata die armenische Edition, oder besser ihr Prototyp, ein getreues Abbild des Euseb-Originals erster Auflage darstellt. D. h. die erste 
Einleitung. III. Einrichtung u. Textherstellung der vorliegenden Ausgabe XXIII

eusebianische Chronik-Edition zeigte auch in der äußeren Anlage großenteils die beim Armenier überlieferte Gestalt, die sich hinwiederum am meisten der der sog. Familie des Spatium Historicum der Hieron.-Chronik nähert. Mit den Schoeneschen Ausführungen betr. die Hieronymus-Chronik, bzw. ihr Verhältnis zum euseb. Original stimme ich wesentlich überein, mit folgender Ausnahme jedoch: daß nämlich, nicht bloß dem Texte nach, sondern auch in der äußeren tabellarischen Anlage und in der Disposition der Lemmata die armenische Edition ein getreues Abbild des Eusebius-Originals erster Auflage darstellt. Nicht der Armenier erst hat die Verteilung der Lemmata auf zwei Randcolumnen geschaffen, sondern diese Anordnung ist als ursprüngliche auf Eusebius selbst, bzw. auf seine erste ChronikAusgabe zurückzuführen. Ebenso ist die Voranstellung der Columne des Hebräer-Reiches in dem Zahlengerüste, gegenüber Hieronymus, wo zuerst Assyr. steht, dann Hebr. folgt, als ursprünglich eusebisch zu vindicieren.

Wenn so für die erste eusebische Originalausgabe des Kanons der eminent kirchengeschichtliche, unter christlich-pragmatischer Geschichtsauffassung entstandene Charakter des Kanons äußerlich durch die Voranstellung des Hebräer-Filum vereint mit der am linken Rande sich anschließenden Textcolumne der Bibel- oder Hebräergeschichte zum Ausdruck kommt, so ist ebenso bezeichnend für den mehr weltlichprofan-universalgeschichtlichen Charakter der zweiten Ausgabe die Verdrängung der Hebräer aus der I. Reihe und ihre Ersetzung durch das Filum Assyriorum, das nun die Reihe der verticalen Zahlencolumnen einleitet. Infolgedessen wurde auch die ursprünglich am linken Rande stehende Textcolumne der Hebräer, deren Datierung an dem eigenen filum regni durch das Zwischentreten der Assyrerreihe gestört war, entsprechend umgesetzt, und zwar folgte sie ihrem filum regni nach rechts, d. h. sie wurde dem filum Hebraeorum an rechter Seite zwischen den Zahlentabellen angereiht. Weiter wurde dann, teils aus Gründen der räumlichen Symmetrie in der Anlage, teils aber insbesondere, um eine genauere Datierung der einzelnen Lemmata an ihren bezüglichen fila regni zu ermöglichen, da die überwiegende Zahl der Lemmata sich inhaltlich auf die hellenistisch-römische Geschichte bezog, deren Zahlenfila von ihren im rechtsrandigen Spatium historicum befindlichen Lemmaten über den weitaus größten Teil des Kanon hin durch das störende, übrigens fast historienlose filum regni Aegyptiorum getrennt war, auch die Versetzung der rechten Textcolumne vom Rande zwischen die verticalen Zahlenreihen vollzogen, derart, daß in der Regel die nunmehrige interfilare Textcolumne sich rechts an die fila der Griechen- 
resp. hellenistisch-römischen Dynastien anreiht, während das filum regni Aegyptiorum als gleichsam unnützes für die historischen Textdata nach rechts abgeschoben wird'.

Deutlich von den Randlemmata geschieden und meist auch durch stärkere Schrift hervorgehoben sind in unseren Codices gewisse quer über die Seite durchgeschriebenen Textabschnitte, Perikopen oder Periochen, die ihrem Inhalt nach die Hauptstadien und Epochen der chronologischen Zählung bezeichnen, indem sie an weltgeschichtliche Daten und Begebenheiten anknüpfend gleichsam feste Ruhepunkte und Recapitulationen der bisherigen synchronistischen Zählperioden aufstellen und so den Kanon in verschiedene, auch rein äußerlich durch große Titelrubriken wie die von der Einnahme Ilions, von der Zerstörung des jerusalem. Tempels, gekennzeichnete Abschnitte einteilen.

Verschieden von diesen Haupt- oder epochalen Quertextabschnitten ist eine dritte Klasse von Lemmaten, die ebenfalls quer über die ganze Seite geschrieben werden; deren secundäre Bedeutung aber schon graphisch daraus erhellt, daß für sie das Liniengerüst nicht pausiert, sie vielmehr über das fertige verticale Liniensystem quer übergeschrieben werden. Schon der Umstand, daß diese Querlemmata einem bestimmten Zeitraum, dem der Kaiserperiode und zugleich des Christentums eigen sind, weist den Gedanken ab, als ob diese Disposition lediglich eine willkürliche, durch raumökonomische Rücksichten bedingte sei. Auch stellt sich bei näherer Betrachtung heraus, daß nicht sowohl Raumrücksichten oder gar Copistenwillkür, als vielmehr sachliche Gründe bestimmend für diese Lemma-Stellnng gewesen sind. Die als Querlemmata überlieferten Stücke beschränken sich nämlich wesentlich auf folgende Punkte:

1) vor allem die Berichte von den Christenverfolgungen, die sich gleichsam als Gegenstück bzw. Fortsetzung an die vorherigen und gleichzeitigen Judenverfolgungen anreihen;

1) Außerdem spielt indessen in der Stellung der Textcolumnen $z u$ den einzelnen fila regnorum bei dieser Utherlieferungssippe das Raumprincip der Symmetrie eine offenbare, wenn auch secundäre Rolle; im einzelnen ist über die wechselnde Stellung zu bemerken:

a) Zuerst geht die rechte Textcolumne der letzten Zahlenreihe voran;

b) Mit Zunahme der Anzahl der Regna-fila, die sich schließlich bis auf 9 steigern, rückt die rechte Textcolumne eine Stelle nach links, wird also vor das vorletzte Filum gestellt;

c) Bei abermaliger Abnahme der Columnen der Fila Regnorum tritt die rechte Textcolumne wieder vor die letzte Fila-Columne. - Es soll eben der răumliche Abstand zwischen der linken und der rechten Textcolumne möglichst constant gewahrt bleiben. 
Einleitung. III. Einrichtung u. Textherstellung der vorliegenden Ausgabe $\mathrm{XXV}$

2) die Bischofsliste der Jerusalemischen Kirche.

Daß diese Anordnung der Lemmata etwa das eigenmächtige Werk des armenischen Übersetzers sei, halte ich für ausgeschlossen. Alles deutet darauf hin, daß er sie schon in seiner griech. oder syr. Vorlage vorgefunden habe und zwar als echt eusebianische Anordnung. Hierfür spricht: 1) daß auch in der Überlieferung der Hieronymus-Version die Anni persecutionum teils am Rande, teils aber auch zwischen den Zahlenreihen in den Codd. angebracht worden sind (Schoe. Weltchron. p. 86), wodurch diese Zeitpunkte als wichtigere Epochen, bzw. die entsprechenden Berichte als besonders bedeutsame hervorgehoben werden; 2) daß für Eusebius, den Syro-Palästinenser und Bischof der palästinischen Metropole Cäsarea, gerade die jerusalemische Mutterkirche im Vordergrund stehen und vor allen anderen in Betracht kommen mußte.

$\mathrm{Da}$ übrigens auch, abgesehen von dieser besonderen Partie, dem jeweiligen Raumbedürfnis entsprechend, die Randlemmata, wiewobl ausnahmsweise und selten, in Querlemmata übergeführt werden konnten, wird, wenn auch kaum für unsere armen. Codd., die in ihrem 1. Teile diese Übung vermeiden, so doch für die hieronymische Sippe des Spatium historicum als uralte Übung bezeugt, wo ganz geläufig die als Randlemmata im Spat. hist. begonnenen Textstücke weiter als Querlemmata unter den horizontalen synchronistischen Zahlenreihen ausgeschrieben zu werden pflegen.

Als Mittel zur Datierung der einzelnen Randlemmata verwenden Codd. EG horizontale Linien, die, jedem einzelnen Lemma übergezogen, auf die entsprechenden synchronistischen Horizontal-Jahrreihen der Zahlencolumnen hinweisen, an welche die Lemmata gebunden sind. Diese graphische Datierungsweise ist jedoch nicht consequent durchgeführt. Sie beschränkt sich, analog der im Folgenden noch näher zu erörternden Dekadeneinteilung durch Horizontallinien, wesentlich auf den ersten Teil des Kanons, etwa bis zum Einschnitt von der Einnahme llions. Aber auch innerhalb dieses ersten Teiles ist diese graphische Datierungsweise der Lemmata nicht streng und allgemein eingehalten, indem, anscheinend willkürlich, die horizontalen Marginallinien bald gesetzt, bald ausgelassen werden. Solche horizontale Marginallinien stehen in Cod. E, von welchem $G$ nur unwesentlich abweicht, als Datierungs-Anzeiger zu Rand-Lemmaten an folgenden Stellen:

Cod. p. 110: a. J. Abr. 345, 351, 355, 360 u. 378; p. 111: a. J. Abr. 395, 400, 403; p. 112: a. J. Abr. 424/25, 435; a. J. Amenoph. 25 , a. J. Oros. 4 u. 7/8; p. 113: a. J. Abr. 455; p. 114: a. J. Abr. 465, 468, 475, 476/77; a. J. Achencheres 1, 7, 8/9, 11/12; p. 115: a. J. Abr. Eusebius, Cbron. arm. 
495, 500, 507, 508/9, 511, 517/18; a. J. Acherres 1, 2, 5, 8; p. 116 : a. J. Abr. 529, 531/32; a. J. Egipt. 1; p. 117: a. J. Abr. 550/51, 556/57, 562, 572, 578; a. J. Egipt. 17/18, 26/27, 24/35, 46/47 (neben Pandios); p. 118: a. J. Abr. 582/83, 587/88, 602; a. J. Menophis 1, 9/10; p. 119: a. J. Abr. 613, 617/18, 624/25; a. J. Menoph. 25/26, 31; p. 120: a. J. Abr. 651/52, 656; a. J. Sethos 27/28, 28/29; p. 121: a. J. Abr. 692/93; a. J. Sethos 41/42; p. 123: a. J. Ramps. 65/66; p. 124: a. J. Abr. $789 / 90$, a. J. Amenoph. 13, 38. Von hier ab nur noch vereinzelt p. 126: a. J. Abr. $859 / 60$; p. 127: a. J. Abr. $898 / 99$; p. 130: a. J. Abr. $987 / 88$; p. 133: a. J. Orthon. 2; p. 134: a. J. Abr. 1231/32; p. 135: a. J. Abr. 1258; p. 136: a. J. Abr. 1261/62, 1269/70; p. 137: a. J. Sabak. 8, a. J. Sebichos $4 / 5$. Von hier ab kommen marginale Indexlinien überhaupt nicht mehr vor. In gewissen Fällen, so z. B. a. a. Abr. 499/500 und a. a. Abr. 510/511 stellen sich diese Marginal-Linien einfach als Verlängerung oder Fortsetzung der das Zahlengerüst abteilenden Dekadenund sonstigen Linien dar; und unzweifelhaft hängt die Erscheinung des Marginal-Liniensystems des I. Kanon-Teiles mit der ursprünglichen Dekadeneinteilung der Zifferncolumnen zusammen, so zwar, daß die ursprünglichen Dekaden- bzw. Heptaden- oder Pentaden- u. dgl. Linien sich über das Fila-Columnengerüst in die Ränder des Spatium historicum hinein verlängert haben werden; diese Randverlängerungen mochten als geeignete Indices für die Datierung der Lemmata erscheinen, so daß nachgerade, zumal da nach Zerrüttung des ursprünglichen Dekadensystems die eigentliche Bedeutung der marginalen Linien nicht mehr hervortrat, auf Grund dieses Ansatzes sich eine, wenn auch mangelhaft durchgeführte, graphische Datierungsweise der Randlemmata mittelst horizontaler Linienzeiger ausbildete. Wenn nun auch diese überlieferte Einrichtung der Randlemmata m. E. nicht nur für authentische, sondern auch, bei der conservativen Weise des armen. Übersetzers, für bereits auf seine Quellenvorlagen zurückgehende zu halten ist, so schien es doch ratsam, dieselbe als störendes Beiwerk, wodurch der Text des Spatium historicum in mannigfache, mehr willkürliche als streng rationelle Abschnitte zerteilt worden wäre, in die folgende Edition nicht aufzunehmen, zumal da nach dem vorhin vollzogenen, diplomatisch genauen Vermerk der betreffenden Horizontallinien-Indices von ihrer Reproducierung im Texte füglich abgesehen werden durfte. Statt dessen wird daselbst durch ein Punktierungssystem, wonach jedem Lemma ein bei der entsprechenden Datierungszahl im Liniensystem wiederkehrender Punkt als Index vorgesetzt wird, die Datierungsbeziehung der einzelnen Lemmata zu ihren zugehörigen horizontalen Jahresreihen ausgedrückt.

Ebenso sind in unsere Textausstattung aus Gründen der Ver- 
Einleitung. III. Einrichtung u. Textherstellung der vorliegenden Ausgabe XXVII

einfachung und Entlastung der ohnehin sehr schwierigen typographischen Wiedergabe des Kanons nicht aufgenommen worden die gleichmäßig über den ganzen Kanon hin von den Codices überlieferten äußeren Randleisten, die auf jeder Seite in Form eines regelmäßigen rechteckigen Rahmens einerseits den oberen und unteren Textrand abschließen, anderseits die marginalen Textkolummen nach rechts und links umfassen und zusammenhalten, so daß also die zu einem Ganzen vereinigten Zahlentabellen nebst zugehörigen Textcolumnen sich auch äußerlich in Form von symmetrisch aufgebauten, geschlossenen chronologischen Tafeln als solche darstellen.

Was die Stellung der Lemmata innerhalb der einzelnen Seiten, ob am äußeren oder am inneren Seitenrande, und ihre Verteilung auf die zwei Columnen des spatium historicum anbetrifft, so ist in unser Ausgabe von der überlieferten Anordnung nur ausnahmsweise abgegangen, nämlich in Fällen, wo offensichtlich durch Textverschiebung oder -zerrüttung die Stellungen gewechselt worden sind. Da nun, wie oben bemerkt, im Original-Eusebius die Scheidung herrscht, daß die Columne des linken Seitenrandes so gut wie ausschließlich nur die Tatsachen der heiligen Geschichte, die rechte Columme diejenigen der Profangeschichte enthielt, so haben wir hierin eine Art Kriterium für die Restitution der von der einen in die andere Columne versprengten Lemmata auf ihren ursprünglichen Stand.

So ist denn auch bezüglich der Disposition der einzelnen Lemmata innerhalb ihrer Columnen und ihrer Beziehung und Verbindung zu den entsprechenden Jahren der fila regnorum die überlieferte Stellung in der Regel genau gewahrt, bzw. in Fällen von Dislocation und Verschiebung das ursprüngliche Verhältnis wiederhergestellt worden. Während Ausgabe $\mathrm{P}(\mathrm{A})$ in dieser Hinsicht noch fast ausschließlich auf Codex $\mathrm{G}$ fußt, $\mathrm{Z}$, wiewohl außerdem auch Cod. $\mathrm{N}$ verwertend, durchweg allzu willkürlich vorgeht, wohl unter dem Bestreben der Angleichung an den hieronymianischen Kanon, legen wir der Ansetzung der einzelnen Lemmata und ihrer Beziehung zu den Fila regnorum den zuverlässigeren Cod. E zugrunde; wobei wir durchgängig die handschriftlichen Varianten bzw. divergierenden Dispositionen der bisherigen Ausgaben vermerken.

Die Tabellen der sogg. Fila regnorum betreffend, so waren diese offenbar im Original-Eusebius in Dekaden gegliedert, und zwar durch horizontale Durchschnittslinien, die über den betreffenden Zehnerzahlen einherliefen. Diese ursprüngliche Dekadeneinteilung kommt noch in Cod. arm. zum Durchschein durch die unter folgenden Jahren stehenden Abschnittslinien: a. Abr. 439, 469, 499, 519, 569, 579, 589, 
$599,619,639,689,709,719,759,769,789,849,939,1009,1109,1139$, $1159,1259,1359,1379,1419,1459$ u. a. m. Gleichwohl wäre es zu weit gegangen, nach diesem Schema den armenischen Euseb.-Kanon uniformieren und mit Aucher (Ed. A) die ursprüngliche Dekadeneinteilung, die übrigens, nach der ganzen Anlage des armenischen Kanons zu urteilen, schon früh durch Halbierung in Pentaden modificiert worden sein mag, mittels unter den betreffenden Neunereinheiten eingelegter Linien durchführen zu wollen. Erscheint doch beim Armenier jenes ursprüngliche System durchbrochen durch ein anderes neben jenem einherlaufendes, wonach unter anderen als Intervalle vorkommen: a. Abr. 367-377, 387-395, 597-605, 457-465, 466-475, 426-446 (Doppelintervall), 686-696, 697-705, 727-737 etc. Augenscheinlich greift hier in das ursprüngliche eusebische Dekadensystem eine Zergliederung in Heptaden ein, wie aus folgendem Beispiel hervorgeht: überliefert ist in der graphischen Tabellenanlage noch das Originalintervall 589-599, jedoch durchbrochen durch eine zwischen 596 -597 eingelegte Linie; dadurch entsteht einerseits die Heptade 590-596 nebst der Triade 597-599, anderseits das neue (unvollkommene) Dekadenintervall 597-605. Heptadenabschnitte sind z. B. a. Abr. 440-446, $552-558,590-596,606-612,613-619,651-657,658-664,706-712$, 713-719, 720-726, 738-744, 753-759, 776-782, 783-789, 819-825, 961-967, 1119-1125, 1144-1150,1160-1166, 1241-1247, 1287-1293, 1294-1300, 1321-1327, 1396-1402, 4438-1444, 1460-1466. Daneben die bereits erwähnten Triaden, sowie Sextaden und Tetraden, letztere insbesondere markant hervortretend in Form der Abteilung nach Olympiaden. Man beachte die in der Tabelle gekennzeichneten Abschnitte: a. Abr. 1248-1251 (Ol. 3), 1252-1255 (Ol. 4), 1256-1259 (Ol. 5), 1268-1271 (Ol. 8), 1324-1327 (0l. 22), 1340-1343 (0l. 26), 1360-1363+1364-1367 (Ol. 31-32), 1368-1371 (Ol. 33), 1372-75 +1376-79 (0l. 34-35), 1380-83 (0l. 36), 1384-87 (0l. 37), 1388-91 +1392-95 (Ol. 38-39), 1408-1411 (0l. 43), 1412-15+1416-19 (0l. 44-45), 1420-23 (0l. 46), 1424-1427 (0l. 47), 1428-1431 (0l. 48), 1448-1451+1452-1455 (0l. 53-54), 1456-1459 (Ol. 55). Daß jedoch diesem Teilungsmodus keine eigentliche principielle Bedeutung beizumessen ist, lehrt schon die Ausdehnung desselben auch auf den außerhalb der Olympiadenära liegenden Zeitraum; vgl. die Abschnitte: a. Abr. $466-469,511-514,534-537,686-689,693-696,803-806,807-810$, 908-971, 972-975, 1010-1013. Nicht sowohl in den genannten Systemen als vielmehr in dem Princip der räumlichen Symmetrie liegt der eigentliche Einteilungsgrund des Armeniers, indem schließlich doch dieses Princip bestimmend wirkt für die Anwendung jener, 
Einleitung. III. Einrichtung u. Textherstellung der vorliegenden Ausgabe XXIX

und nach Maßgabe desselben, meist unter Anlehnung an die schon in die Tabellen durch die Regentenrubriken vorgezeichneten Einschnitte, der Redactor ziemlich frei und willkürlich das Liniengerüst nach der einen oder anderen der genannten chronologisehen Zählungsmethoden (Dekaden, Heptaden, Tetraden etc.) einteilt. Dieses lineare System der graphischen Anlage ist übrigens in unsern Handschriften nicht streng auf den ganzen Kanon durchgeführt, sondern nur etwa bis zu Alexander d. Makedonier, a. a. Abr. 1514 oder wenig weiter. Nichts hindert uns indessen an der Annahme, daß die weitere Durchführung desselben ursprünglich vorhanden bzw. beabsichtigt gewesen, daß jedoch, analog wie bei der Rubrik der Jahre ab urbe condita, lediglich durch Copistennachlässigkeit oder Bequemlichkeit, bzw. aus Nichtbeachtung einer etwaigen diesbezüglich am Rande vermerkt gewesenen Anweisung betreffend die graph. Anlage der überlieferte mangelhafte Zustand hervorgegangen sei. Jedenfalls geht diese Anlage wesentlich als authentische auf den armenischen Übersetzer zurück und wird, wiewohl etliche Neuerungen und Modificierungen im angedeuteten Sinne von späteren Copisten herrühren mögen, in unserer Darstellung des Kanons getreu wiedergegeben werden.

2. Inhaltlich adäquat, d. h. eine getreu den Sinn, den der armenische Interpret in seine Version gelegt, wiedergebende, auch da, wo diese vom griechischen Original offenkundig oder mutmaßlich abweicht, in welchen Fällen regelmäßig in Form von Anmerkungen auf die abweichende Originalfassung der griech. Version hingewiesen wird.

Zur adäquaten Wiedergabe der ursprünglichen Fassung von Vers. Arm. gehört als Grundbedingung die Textreconstruction und Sanierung bzw. Ergänzung corrupter und defecter Stellen. Es wird dies in vorliegendem Werke nach allen Regeln gesunder Textkritik in einer Weise ausgeführt, daß, mit Ausnahme der oben aufgezählten, durch Blätterausfall bewirkten größeren Textlücken, deren Reconstruction außerhalb des Rahmens unserer Aufgabe lag ${ }^{1}$, der gesamte Text der Eusebiuschronik armenischer Version hier zum ersten Male auf seine ursprüngliche Form wiederhergestellt erscheint. Es erschien uns dies für unsere Publication grundwesentlich und um so mehr geboten, als

1) Die Restitution dieser ausgefallenen Textpartien läßt sich mit ziemlicher Sicherheit bewerkstelligen auf Grund von 1) Hieronymus' Kanon; 2) den Epitomen der eusebianischen Chronik: Samuel v. Anis Chronik, Epitome Syria, sowie Stephan v. Taron (Weltgeschichte) u. a. m.; 3) Sync. Chronographie, Chron. pasch. etc. Nun haben zwar Aucher sowohl als Zohrab-Mai diese Reconstruction vorgenommen; ihre diesbezüglichen Textergänzungen sind jedoch keineswegs definitiv oder philologisch abschließend und bedürfen mindestens einer kritischen Revision. 
die Textüberlieferung dieses Werkes so ziemlich im Argen liegt und auch Petermann, trotz mehrfacher glücklicher Emendationen, sich auf eine systematische Textkritik nicht eingelassen hat, so daß die bislang recipierten corrupten Lectionen vielfach irreführend sein und die armenische Version in falschem Lichte erscheinen lassen mußten. In dem diesbezüglichen, für den I. Teil unter den Text aufgenommenen, für den Kanon als 'Kritischer Anhang" gebrachten Apparatus criticus, der zur Erklärung und Begründung schwieriger Textstellen und Emendationen seine Ergänzung in den am Ende des Gesamtwerkes stehenden 'Zusatzbemerkungen \& findet, dürfte zugleich gewissermaßen die wesentlichste Vorarbeit zu der hoffentlich in nächster Zukunft zur Verwirklichung gelangenden endgiltigen kritischen Textedition der armenischen Eusebius-Chronik geschaffen sein.

Bei der entscheidenden Wichtigkeit, die der armenischen EusebVersion für eine Wiederherstellung der griechischen Original-Chronik zukommt, erübrigt hier noch, zur Ergänzung des unter 2) über inhaltlich adäquate Wiedergabe Erörterten, die diesbezüglich schon im vorigen aufgeworfene Frage nach dem eigentlichen Umfang der Chronik näher zu prüfen und einer Lösung entgegenzuführen. Daß, abgesehen von den vielfachen kleineren Textdefecten, namentlich die Anfangspartie nebst zwei größeren Stücken von je 2 Seiten Text innerhalb der Chronici Canones beim Armenier ausgefallen sind, steht sicher und ist bereits erwähnt worden. Wie aber steht es mit dem Abschluß des I. Buches, der eigentlichen Chronographie? Wo endigte der I. Teil der Euseb-Chronik? Hat Euseb die im Prooemium versprochene Consularliste wirklich auch gegeben?

Der Umstand, daß er gegen Schluß des Römer-Abschnittes nach dem Dionys-Citat es geradezu ablehnt, die Consuln im einzelnen aufzuführen, läßt vermuten, daß Euseb den in dem Prooemium gefaßten diesbezüglichen Plan im Laufe der Arbeit fallen gelassen habe bezüglich der Consuln der Republik bis auf Jul. Caesar. Oder auch: er wollte sich vorbehalten, diese Consularliste als besonderen Appendix zu dem Werke zu liefern, wozu er aber nicht mehr gekommen wäre.

Als integrierender Bestandteil der Chronik sollte aber wenigstens die in die Kaisergeschichte verwobene Consularliste der Kaiserzeit erwartet werden, nach dem diesbezüglichen Einführungssatze am Schlusse des Römer-Abschnittes.

Dieser Schlußteil aber fehlt, das I. Buch der Chronik bricht mit jenem Satze ab, der die Disposition zu eben diesem zu erwartenden Schlußteil enthält. 
Einleitung. III. Einrichtung $u$. Textherstellung der vorliegenden Ausgabe XXXI

Bisher neigte man zu der Annahme, Euseb habe wirklich noch dảs nach dieser Disposition zu erwartende Capitel, die Behandlung der Chronologie der Kaiserzeit, verfaßt und damit den I. Teil der Chronik beschlossen; durch verwahrloste Überlieferung sei diese Partie verloren gegangen. Ich glaube jedoch annehmen zu müssen, daß die Chronik in diesem Sinne nie vollendet worden, daß vielmehr Euseb nicht über den uns vorliegenden Abschluß der armenischen Version hinausgekommen ist. Ich nehme an: mit Erreichung ${ }^{\circ}$ der römischen Kaiserzeit sieht der Verfasser den ersten Hauptteil der Aufgabe seines Werkes, die Darstellung der Chronologie der vorchristlichen Zeit gelöst. Mit Augustus und dem Auftreten des Christentums beginnt für ihn eine neue weltgeschichtliche Epoche, deren Behandlung als zugleich kirchengeschichtliche sich gleichsam als zweites ebenbürtiges Hauptstück an das uns vorliegend überlieferte I. Buch der Chronik anzuschließen hätte. Zur chronologischen Darstellung dieser halb kaiser-, halb kirchengeschichtlichen Periode ist es aber nicht gekommen, vielleicht bewußterweise und nicht ohne wohlerwogene Berechnung: denn hier tritt ja desselben Autors Kirchengeschichte als glänzendes Ersatzstück für diese Periode ein, mit Hinweis auf welches eine weitere eingehendere chronologische Behandlung der Kaiserzeit gewissermaßen überflüssig erscheinen mochte.

Hierfür spricht auch der Umstand, daß Eusebs Weltchronik jedenfalls, ihrer Verfassung nach, seiner Kirchengeschichte vorangegangen ist, da sie Hist. eccl. I 1, 7 zitiert wird (Schoe. Weltchron. p. 259); dies gilt wenigstens sicher für die erste Ausgabe der euseb. Chronik, d. h. wie hier mit Verweisung auf die nachfolgenden Ausführungen vorweggenommen sei, eben diejenige, die als Quellenvorlage der armenischen Version gedient hat. Mit Rücksicht auf diese in Sicht gehabte, bevorstehende Publication der Kirchengeschichte scheint Euseb die Einzelausarbeitung der Chronographie des kaiserlichen $\mathrm{d}$. h. christlichen Roms zunächst hinausgeschoben zu haben und schließlich davon, mit Hinweis auf dieselbe Hist. Eccl., ganz abgestanden zu sein, wobei für den Kirchenvater und Kirchenhistoriker Euseb schließlich auch das Moment von entscheidender Bestimmung gewesen sein mag, daß eine detaillierte chronographische Darstellung der röm. Kaiser- bzw. christlichen Periode durch die Chronik ja eben der Materie seiner Kirchengeschichte vorgegriffen hätte, deren Erfolg dadurch schwer beeinträchtigt gewesen sein würde, da ihr dadurch der Reiz des Neuen und der Anspruch auf volle Originalität benommen gewesen wäre. Um also nicht nur unnütze, sondern geradezu nachteilige Wiederholung derselben Materie zu vermeiden, beschränkt sich Euseb darauf, die chronographische Darstellung 
der fraglichen Periode nur seiner Kirchengeschichte, d. i. demjenigen Werke, dem sie unentbehrlich war, einzuverleiben und mit dieser zu verweben; diesem seinem Haupt- und Lieblingswerk opfert er gewissermaßen einen Teil der ursprünglichen Disposition der Chronik, die er jener zuliebe für die fragliche Periode der Kaiser unvollendet gelassen hat.

Ein weiterer Ersatz für die vermißte Chronologie der Kaiserzeit ist übrigens geboten im Schlußteile des Kanons, der bezeichnenderweise der kirchlichen Chronologie dieser Zeit ein nicht minder eingehendes Interesse widmet als der politischen.

Ich schließe also, daß Euseb den I. Teil der Chronik nach der in dem Proömium gegebenen vollen Disposition überhaupt nie ausgeführt hat; sich vielmehr mit der Zeichnung der wichtigsten Partien begnügte und für den Schluß, die Chronologie der Kaiserzeit, gleichsam im Hinweis auf seinen Kanon sowie auf seine Kirchengeschichte die Detailausarbeitung zunächst verschob oder sich auf später vorbehielt, und schließlich ganz fallen ließ.

Zur Bestätigung dieses Ergebnisses läßt sich anführen, daß ebenso in der hauptsächlich auf dem armenischen Euseb fußenden Chronik des Samuel von Ani derselbe Zeitraum kaum gestreift wird. Das Samuelsche Chronik-Werk beschränkt sich auf eine summarische Darstellung der Listen der Latiner-Könige sowie der altrömischen Könige, die aus dem Schlußteil des Abschnittes 'Römer-Chronographies des I. Buches unser Chronik excerpiert sind. Hier wie in unserer Chronik schließt die eigentliche Darstellung der Römer-Chronographie mit Julios Kaisar und Augostos ab. Von einer Consularliste der Kaiserzeit oder dgl. mehr, was der eusebischen Disposition bezgl. sRömers entspräche, ist auch hier keine Spur vorhanden. Da sonst im allgemeinen der Chronograph Samuel Aniensis der Euseb-Chronik in den wesentlichsten Grundzügen genau zu folgen pflegt, so hätte er, falls er dergleichen für die Kaisergeschichte vorgefunden hätte, es sicher nicht übergangen, sondern im Auszuge resümiert. Da nun aber diese seine Chronographie im I. Teile die Römerzeit gleichwie bei unserm Euseb mit dem Ende der Republik abbricht und nur bis zum ersten Kaiser führt ${ }^{1}$, so folgt

1) Die im zweiten, mit der christl. Zeit anhebenden synchronistischen Teile der Samuel-Chronik (Ed. pp. 52 sqq.) parallel zu den Regna Judaeorum, Persarum und Armenorum gegebene Darstellung der römischen Kaiser ist bis zur Regierung Konstantins d. Gr. weiter nichts als ein getreuer Auszug aus der armenischen Kanon-Version des Eusebius. - Wollte man gleichwohl, trotz aller entgegenstehenden Unwahrscheinlichkeit, wirklich den Ausfall eines ursprünglich eusebi- 
Einleitung. III. Finrichtung $\mathrm{u}$. Textherstellung der vorliegenden Ausgabe XXXIII

hieraus, daß die seinem Werke als Quelle zugrunde gelegte, sicher nicht verstümmelte, sondern den unversehrten, ursprünglichen Umfang zeigende armen. Chronik-Version, ebensowenig wie unsere überlieferte Fassung eine besondere Behandlung der Kaisergeschichte am Schlusse vom Buch I enthielt, und daß mithin das I. Buch der Chronik in seiner vorliegenden Fassung in vollem, ursprünglichem Umfang überliefert ist.

Was schließlich das Endjahr der armenischen Chronik betrifft, so durfte nach dem Stande der Überlieferung mit Bezug auf Chron. I p. 62, 4, wo als Endtermin der synchronistischen Zählung die Vicennalia Constantini angegeben werden, für die folgende Editio füglich angenommen werden, daß der armenische Kanon wirklich bis zu diesem Termin gereicht habe und daß lediglich durch zufällige Textverstümmelung der Schluß der armen. Version verloren gegangen sei. Hierfür spricht insbesondere noch, daß auch die der Hieronymus-Chronik zugrunde gelegte griechische Version nach dem ausdrücklichen Zeugnis von Hier. Can. a. a. Abr. 2343 mit den Vicennalia abschloß.

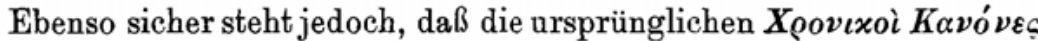
des Euseb die Zeit Constantins nicht erreicht haben. Und da dürfte denn der Abschluß unsrer Überlieferung auf das Jahr Diocletian. 16 nicht zufällig, sondern vielmehr zu vermuten sein, daß die vorliegende Überlieferung des Armeniers, der bekanntlich, im Gegensatz zur Versio Hieronymiana, auf die erste eusebische Originalausgabe zurückgeht, den vollen Textumfang jener ersten Originalfassung unversehrt wiedergibt, die demnach auf das 16. Reg.-Jahr Diocletians ihren Abschluß gefunden hätte. Insofern jedoch, nach dem unten noch näher zu Erörternden, dieselbe armen. Version eine secundäre Überarbeitung nach der II. emendierten Chronik-Ausgabe Eusebs erfahren hat, welch letztere bestimmt bis zu den Vicennalia Const. erweitert war, darf füglich, neben einer älteren, kürzeren armenischen Fassung als Repräsentant von Editio orig. I, noch eine jüngere, bis zum J. 20 Constantins geführte, als Repräsentant von Editio orig. II angenommen werden.

schen Abschnitts römischer Kaiser-Chronographie am Schluß von Bd. I annehmen, so dürften füglich in den diesbezüglichen Tabellen des Kanons, zumal auch in den diesem beigegebenen außerordentlich reichen Textstücken, sich Anhaltspunkte zu dessen Reconstruction bieten. 


\section{Verbreitung und Benutzung der armenischen Eusebius-Chronik.}

"Jeweils sind es die Scharfsinnigsten und Weisesten der Männer gewesen, die in ihrem Volke die Zeitbeschreibung darzustellen unternommen haben. In diesem Betreff ist als Berühmtester über die große Mehrzahl hervorragend geworden Eusebius der Selige unter den Früheren und den Späteren" (Sam. An. Chron. Prolog). Mit diesen Worten, worin sich das Urteil der Zeitgenossen, des XII. Jhd.s, widerspiegelt, leitet der armenische Chronograph Samuel von Ani sein Werk ein, das sich hauptsächlich als eine gedrängte Epitome aus der Chronik des Eusebius unter Mitbenutzung des Historikers Moses von Choren für die armenische Geschichte ausdrücklich darstellt: ১Unternehmen werden wir zu schreiben aus zuverlässigen Schrifturkunden nach Eusebius' Chronischen Kanones und Moses, dem Geschichtschreiber der Armenier (Sam. An. Chron. Text) ${ }^{1}$. Die Samuelische Chronik bedeutet gleichsam den Schluß- und Markstein für die traditionelle Würdigung und Wertung der EusebiusChronik auf armenischem Boden, welche sich bis in weit frühere Jahrhunderte zurückverfolgen läßt. "Samuel von Ani (Gelzer, Sext. Jul. Afr. II 475) schließt sich in der Teilung seines Werkes in eine Chronik und einen Kanon an Eusebius an und geht nur zum Teile auf die Alexandriner zurück.s Das heißt nach mir: Sam. v. Ani benutzt als Hauptquelle den Eusebius, nebenbei aber auch die alexandrinischen Chroniken des Panodoros und des Anianos (um 410) bzw. deren Überarbeitungen. Im XI. Jahrhundert ist es der Polyhistor Gregor Magistros Pahlavuni, der die eusebische Chronik benützt und vielfach stellenweise anführt. Weiter zurück sodann, im X. Jahrhundert, der Historiker Stephan von Taron (Asolik), der für seine Weltgeschichte den Euseb als Hauptquelle zugrunde legt, nicht nur stofflich, sondern teilweise auch nahezu im Wortlaute, indem er große Auszüge aus demselben seinem Werke einverleibt. Die Bedeutung, die der EusebiusChronik als Quelle dieses Werkes eingeräumt wird, kommt zum Aus-

1) Sam. Anien. Chron. lateinisch ediert als Anhang der lat. Eusebius-Edition von Zohrab-Mai 1818-1839; der Kanon allein bei Brosset, Collection d'hist. Armén. St. Pétersbourg 1876, II 340-483 (in französ. Ũbersetzung); im Excerpt bei Dulaurier, Histor. des Croisades I 447-468; Armenische Ausgabe von Aršak TêrMikhelian, Vagharšapat 1893. 
Einleitung. IV. Verbreitung u. Benutzung der armen. Euseb.-Chronik XXXV

druck in der im Prolog desselben getanen Erwähnung: sSo haben denn die geistig vorzugsweise ausgezeichneten Denker ........ in Schriftelementen geschichtlich verzeichnet die Namen und die Zeiten der Könige und sämtliche in der Zeit geschehenen, des Gedächtnisses würdigen Taten. So jener wahrhafte Berechner der Zeiten, Eusebius Pamphili, welcher beginnend mit dem ersten Menschen, da wo er aus dem Orte der Wonne heraus verwiesen wird, sein Thema auf die Vicennalien Konstantins, des großen Königs, beschließt (Steph. Tar. hist. univ. Prol.). Ausgangs des IX. Jahrhunderts finden wir Thomas den Artzrunier, der in seiner Stammgeschichte durchgehends aus der Chronik des Eusebius als seiner Hauptquelle schöpft, mitunter wortgetreu excerpierend und besonders den Kanon verwertend. Ohne im einzelnen auf sämtliche übrigen Vertreter derselben ununterbrochen fortlaufenden historischen Tradition näher eingehen zu wollen, auf den Historiographen Johannes VI. Katholikos aus dem IX. Jhd., der dasselbe chronographische Werk excerpiert, auf Zacharias I. Katholikos aus dem IX. Jhd., der es in seinen Homilien erwähnt, und noch andere mehr, beschränken wir uns lediglich darauf, den frühesten Spuren der armenischen Version des Eusebischen Werkes nachzugehen. Sie lassen sich nachweisen bei Lazar von Pharpi und Moses von Choren: bei Lazar von Pharpi, der im Proœmium seines Geschichtswerkes unverkennbar auf das Proœmium der Eusebius-Chronik Bezug nimmt, und zwar teilweise in der Originalfassung und mit Wiederholung des armeno-eusebianischen Wortlautes ${ }^{1}$; bei Moses von Choren, in dessen Geschichte sich deutliche Spuren derselben Chronik zeigen ${ }^{2}$. Bereits Aucher ${ }^{3}$ und Saint-Martin ${ }^{4}$ haben auf

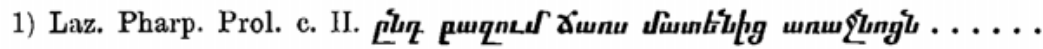
whyg sdurchgangen habe ich zahlreiche Bücherabhandlungen der Altvorderen . . . . Vgl. Euseb. Chron. Prolog.

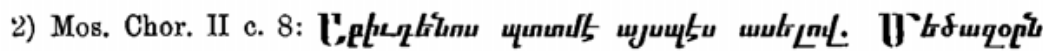

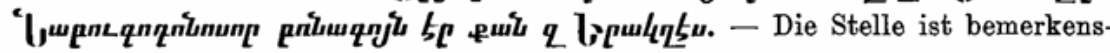
wert dadurch, daß sie das Abydenosfragment ganz in der überlieferten eusebianischen Fassung wiedergibt, mit Umsetzung des Autornamens Megasthenes in das

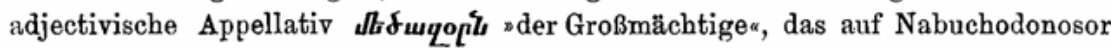
bezogen wird, ganz wie in unserer Euseb-Version. Als Beispiel der zahlreichen Anklänge an den Euseb sei hervorgehoben die Phrase: ‘Luy etrq $\boldsymbol{b}$ เ

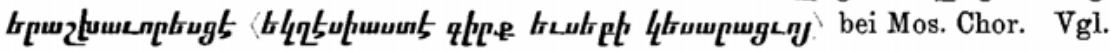
dieselbe Phrase Euseb. Chron. Ed. A. I p. 90, II p. 11 .

3) Ed. A. p. VIII.

4) Journal des Savans 1820 p. 98-99. 
einschlägige Eusebius-Citate bzw. Eusebius-Anklänge bei Moses Chor. aufmerksam gemacht. Die Tatsache steht unleugbar fest.

\section{Die Person des Übersetzers oder Bearbeiters.}

Nicht ohne weiteres als richtig anzunehmen sind dagegen die Folgerungen, die bisher an diese Tatsachen geknüpft worden sind. Zunächst läßt sich trotz der genannten sprachlichen und sachlichen Anklänge keineswegs mit Sicherheit erweisen, daß wirklich der armenische Herodot auch der Urheber der armenischen Übertragung des bedeutendsten altchristlichen Chronographen gewesen sei; eine Vermutung, die zuerst von Aucher als naheliegende ausgesprochen worden ${ }^{1}$, und zu der dann auch Saint-Martin vollends hinneigte. Die Anklänge, so auffallend sie auch sein mögen, bilden an sich noch keinen zwingenden Beweis für die Autorschaft der Eusebius-Chronik armenischer Version durch Moses von Choren, sondern können sehr wohl teils auf Excerpierung, teils auf eingehenderem Quellenstudium und historischer Betrachtung der Eusebius-Chronik beruhen. Aber auch angenommen daß jene Hypothese zutreffend sei, wie ihr denn in der Tat ein hoher Grad von Wahrscheinlichkeit nicht abgesprochen werden kann, so ist doch die dadurch bedingte Ansetzung der Zeit der Abfassung der armenischen Version in entschieden irriger Weise vorgenommen worden.

\section{Die Zeit der Abfassung.}

Von der einseitigen, unzutreffenden Auffassung ausgehend, daß sämtliche einigermaßen ansehnlichere Producte der altarmenischen Literatur, zumal die bedeutenderen Vertreter der Übersetzungsliteratur, dem sog. „Goldenen Zeitalter`, dem der sHeiligen Übersetzer ihrer Schule angehören müssen, haben die ersten Herausgeber, Aucher und Zohrab, als außerhalb jeden Zweifels stehenden den Grundsatz aufgestellt, daß die Entstehung der armenischen Chronik-Version in die älteste Zeit der armen. Literatur, d. h. in das fünfte Jhd., wenn gar nicht schon früher zu verlegen sei. Die hierfür beigebrachten Gründe sind indessen nicht stichhaltig. Zunächst ist der Umstand, daß die Kirchengeschichte des Eusebius schon im V. Jhd. ins Armenische über-

1) Ed. A. p. VI sqq.

2) Journal des Savants 1820 p. 98 sqq. 
setzt worden, keineswegs ein Beweis, daß auch die Chronik desselben schon zur selben Zeit ins Armenische hätte Eingang finden müssen. Auch die für jenes hohe Alter ins Feld geführten ${ }^{1}$ Argumente archaistisch-linguistischer Natur sind nur Scheinargumente; denn manche der von den ersten Editoren infolge ihrer Dunkelheit als Archaismen betrachteten Ausdrücke lösen sich einfach als Corruptelen in gemeingeläufige auf; die im emendierten Texte aber restierende Sprache ist keineswegs so altertümlich, daß sie nicht ebensowohl dem VII.-VIII. oder dem IV.-V. Jhd. angehören könnte. Als entscheidenden Grund für das hohe Alter der armenischen Chronik führt A. ${ }^{2}$ das Zeugnis des Moses von Choren samt dem des etwa gleichzeitigen Lazar v. Pharpi an. Da nun aber, wie durch die Ergebnisse der neueren Forschung untrüglich dargetan ist, der Historiker Moses von Choren nicht (wie nach der einheimischen armen. Tradition angenommen wird) ins fünfte oder gar vierte Jhd., sondern ins VII.-VIII. Jhd. gehört und auch der zweite Gewährsmann, der Historiker Lazar v. Pharpi, entschieden ins VII. oder doch Ende des VI.-VII. Jhds. zu setzen ist - wenn überhaupt jenes Proœmium bei Lazar echt und nicht vielmehr unauthentisch und interpoliert ist -, so ist entgegen der bisherigen beliebten Weise vielmehr zu folgern: die ersten sicher nachweisbaren Spuren der armenischen Version der Eusebius-Chronik finden sich bei Moses von Choren und dessen älterem Zeitgenossen Lazar v. Pharpi, von denen jener ins VII.-VIII., dieser in den Beginn des VII. Jahrhunderts gehört. Da in der dem VII. Jahrhundert vorangehenden Literatur sich nirgends die geringste Spur eines Anklanges an die eusebische Chronik vorfindet, eine etwa früher schon existierende armenische EusebiusChronik aber doch sicher nicht spurlos und ohne Zeichen ibres Einflusses auf die zeitgenössische Literatur, zumal die historische, geblieben wäre, so ist anzunehmen, daß das Alter der armenischen Version nicht viel über das VII. Jahrhundert hinaufzurücken ist, und dürfen wir als Terminus post quem für die Entstehung derselben die letzten Jahrzehnte des VI. Jahrhunderts aufstellen.

Hiermit soll jedoch noch nicht gesagt sein, daß die armenische Fassung der Eusebius-Chronik in der uns überlieferten Form in jener Zeit, d. h. im VI.-VII. Jahrhundert entstanden sei. Es fragt sich vielmehr: ist unsere Fassung der Chronik dieselbe, die dem Moses von Choren vorgelegen hat und die wir als die ältere, ursprünglichere anerkennen

1) Ed. A. l.c.

2) Ed. A. 1. c. 
müssen? Oder aber ist es eine nicht ursprüngliche, überarbeitete Recension, oder gar ein uneinheitliches Werk, eine Contamination aus zwei oder mehreren Versionen, die etwa aus verschiedenen Zeiten und von verschiedenen Übersetzern herrühren und schließlich unter irgend einem Gesichtspunkte zu einer Einheit, zu der uns überlieferten Fassung der Chronik, verbunden worden sind? Dieses führt uns zu näherem Eingehen auf die Entstehungsgeschichte der uns überkommmenen armenischen Version der Eusebius-Chronik.

\section{Der griechische Archetypus als Quellenvorlage des Armeniers.}

sDie Eusebius-Chronik ist im fünften nachchristlichen Jahrhundert aus griechischem Archetypus in's Armenische übertragen worden und in jener ursprünglichen Übersetzungsform auf uns gekommen in der uns überlieferten Version. 8 Diese von Zohrab und Aucher ${ }^{1}$ aufgestellte Lehre wurde zuerst von dem Orientalisten Petermann angefochten ${ }^{2}$, der an ihre Stelle seine eigene Auffassung setzte, die er also formulierte: -Die uns überlieferte Version der Eusebiuschronik ist kein einheitliches Werk, sondern eine Contamination verschiedener, und zwar wenigstens zweier Autoren-Interpretationen, beide aus dem V. Jahrhundert, deren eine aus dem griechischen Archetypus, die andere aus einer syrischen Version geflossen ists. Als wahrscheinliche Zeit der Contamination jener beiden armenischen Versionen zu dem uns vorliegenden Werke vermutet P. das 7. oder 8. Jahrhundert.

Hiergegen wandte sich A. v. Gutschmid, der in einer Recension von Schoenes Ausgabe der Chronik des Eusebius ${ }^{3}$ die Unabhängigkeit der uns überlieferten armenischen Version von der syrischen Übersetzung darzutun suchte.

Untersuchen wir darnach diese wichtige Streitfrage und wenden wir uns zu diesem Behufe zur Betrachtung der sprachlichen Seite des armenischen Chronik-Textes, zunächst und vor allem zu Teil I, der eigentlichen Chronographie, so zeigt dieser Text in dieser Hinsicht einen gemischten Charakter: einerseits ein nationalarmenisches Grundgepräge

1) Vgl. Ed. Z. u. A. Praef.

2) Ed. Schoe. II p. LIII und p. LV.

3) Jahrbücher für class. Philologie 1867 (S. 677-688) und Lit. Centr.-Bl. 1876 S. $885-888$ (K1. Schr. I 422-447). 
Einleitung. VII. Der griech. Archetypus als Quellenvorlage des Armeniers XXXIX

mit leisen Anklängen und Annäherungen an das Syrische, anderseits ein starkes griechisches Element als Einschlag.

Nationalarmenisch ist die Ersetzung von griechischen Götter-, Heroen- und Fürsten-Namen durch armenische: Zeus wird zu Aramazd, Aphrodite zu Anahit, Semiramis zu Śamiram, Arbakes zu Varbakes, Astyages zu Aždahak, Dareios zu Dareh, Hystaspes zu Vyštasp, Artaxerxes zu Artašes; nationalarmenisch die Umsetzung fremder Nomina geographica in einheimische bzw. die dem armenischen Sprachgebrauche

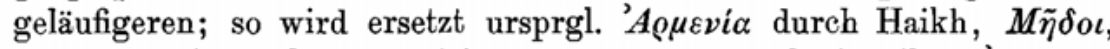

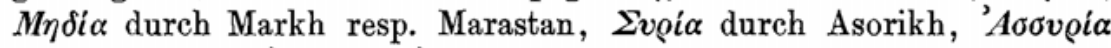

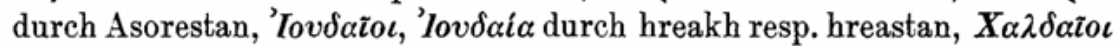

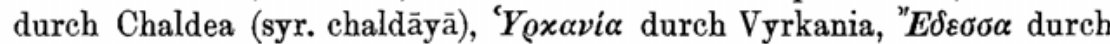

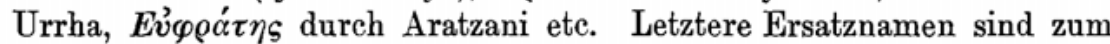
Teil syrische oder syrisierte bzw. vom Syrischen beeinflußte; so z. B. auch Mcbin (Mytsbin) (aus syr. Neșiwin) $=N i \sigma \iota \beta \iota$, šar (assyr. Lw.) ${ }^{1}$ =ó́@os.

Daraus ist natürlich nicht etwa zu folgern, daß der armenische Interpret diese Ausdrücke aus einer syrischen Vorlage entnommen habe; die syrischen Namen und Wörter im I. Buche der Chronik sind in der Regel keine Transcriptionen aus einer syrischen Eusebius-Vorlage, sondern sind als bereits in der Sprache vorgefundenes, längst recipiertes und ererbtes Lehngut zum Ersatz der entsprechenden griechischen Originaltermini herangezogen worden, die noch ganz als Fremdwörter gefühlt wurden, während die syrischen Äquivalente durch Reception in die Bibelsprache bereits eingebürgert waren. So finden wir denn in Chron. I übereinstimmend mit Bibelversion die syr.-armenischen Termini: Śemavon ${ }^{2}$ st. Simeon, Ezr st. Esdras, Ninve st. Ninevi, Dyklath st. Tigris, Šauš (Šôš) st. Susa, Śamrin st. Samaria, Śamyrtaçi ${ }^{3}$ st. Samaritanos u. a. m.

Dagegen sind die außerhalb des armenischen Kulturkreises liegenden Eigennamen regelmäßig in ihrer Originalform, d. h. der griechischen, beibehalten. Gerade in diesem Punkte entfaltet der Armenier, entsprechend übrigens der allgemeinen Gepflogenheit der altarmenischen Übersetzungsschulen, eine ungemeine philologische Akribie, indem er

1) = babyl. šar (Hübschmann, Arm. Gramm. 313); das armenische Wort ist sicher nicht aus gr. $\sigma \alpha \dot{\alpha} \varrho o \varsigma$ entlehnt, wie Brockelmann ZDMG 47, 24 meint. Das Umgekehrte ist das Richtige.

2) Bezeichnenderweise ist jedoch in T. I die griechische Form Simon die vorherrschende, während in T. II (Kanon) stets nur das syrische Šemavon steht.

3) Hybride Mischform aus syr. šamrāyā und gr. $\Sigma \alpha \mu \alpha \rho \varepsilon i r \eta \varsigma$. 
mit großem Verständnis und systematischer Consequenz nicht nur Laut für Laut, sondern auch die Quantität der einzelnen Laute in ihr entsprechendes armenisches Äquivalent umsetzt.

Freilich tut sich durchweg bei der Transcription der Eigennamen das Streben kund, die fremde Lautform mundgerecht zu machen, sie den armenischen Lautgesetzen anzugleichen, sie gewissermaßen zu armenisieren. Namentlich geschieht dies a) auf dem Wege der Assimilation: so ergeben sich die armeno-gräkischen Formen: Pelesger $=$ Pelasger, Peleponesos $=$ Peloponnesos, mekhena $=\mu \eta \chi \alpha \nu \eta^{\prime}$; b) durch Vokalschwund bzw. Vereinfachung und Reducierung nach bekanntem armenischen Lautgesetze: Sikon = Sikyon, Diphes od. Diphres =Diphyes, Kornthos = Korinthos, Lakedmon=Lakedaemon; c) Dissimilation oder Beseitigung von Doppelconsonanz durch Ausstoßung eines Lautes, z. B. dikator $=$ diktator.

Ein drastischer Fall von Armenisierung ist u. a. Ptlomean st. Ptolomaios (griechische Koine-Form) unter offensichtlicher Anlehnung an armen. ptul (Frucht); analog Triptlomeos st. Triptolemos.

In letzteren Fällen könnte allerdings auch syrischer Einfluß an der Umbildung der ursprgl. Lautform beteiligt und mitwirkend gewesen sein. Gleichwohl wäre es zu weit gegangen, hieraus gleich auf directe Übernahme solcher Wörter aus syrischer Vorlage zu schließen, wie dies P Intr. LIV tut, wo unter anderen die Lautformen Thuestes, Tisaphrenes, Kandoles, Thôris (für Thuôris), Sikon (für Sikyon) aus dèm Syrischen erklärt werden.

Die griechische Vorlage des Armeniers für das erste Buch der Chronographie spiegelt sich deutlich wieder nicht nur in der richtigen Transcription der Originalwörter, sondern auch in der an zahlreichen Stellen hervortretenden verkehrten Auffassung des griechischen Originals und der dementsprechenden Wiedergabe durch Arm. Der-

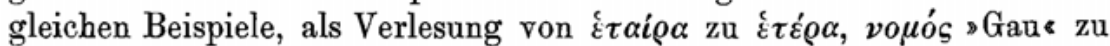

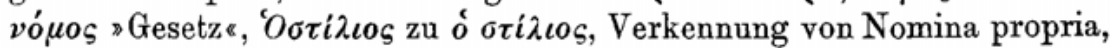

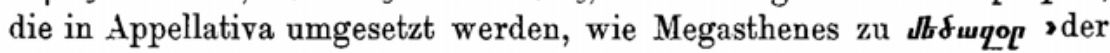

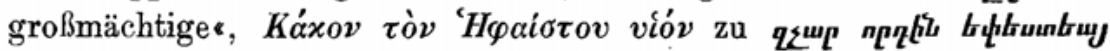
`den bösen Sohn des Hephästos a lassen sich eine ganze Reihe für Buch I aufführen ${ }^{1}$.

Freilich dürften ja allenfalls dergleichen Fehler und Verlesungen des griechischen Originals an und für sich ebensowohl auf Rechnung eines syrischen Interpretators zu setzen sein, der sein griechisches Original falsch aufgefaßt und wiedergegeben, worauf alsdann die also entstandenen

1) Vgl. P (Ed. Schoe. II p. LIV). 
Einleitung. VII. Der griech. Archetypus als Quellenvorlage des Armeniers XLI

ungenauen und falschen Formen und Phrasen durch syrisches Mittel von dem Armenier. übernommen worden wären. Dies mag in der Tat bei dem II. Teil, dem Kanon, der einzige Erklärungsweg sein für Fälle

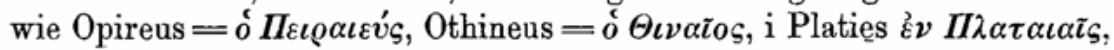
in welchen Fällen der Armenier, wie P. bereits zutreffend bemerkt hat, falls er aus dem Griechischen übersetzt hätte, doch sicher die

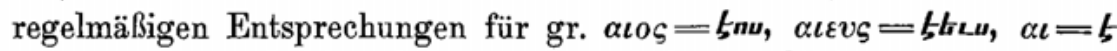
gesetzt und geschrieben haben würde: Opireeus, Othineos, Platees.

Für das I. Buch der Chronik jedoch ist entschieden eine ausgeprägt syrische Beeinflussung nicht vorhanden, wohl aber eine starke griechische Färbung. Diese tritt insbesondere auch hervor in der Nachbildung von

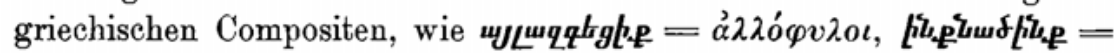

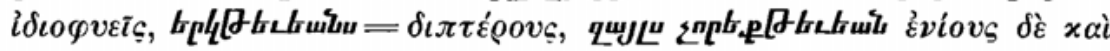

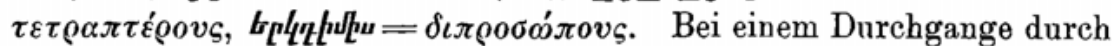
das Syrische, das solche Compositionen nicht zuläßt, sondern in ihre Einzelelemente auflöst, wären diese Ausdrücke vom Armenier sicher nicht durch die betreffenden schwerfälligen Compositen, sondern nach syrischer Art durch je mehrere von einander abhängige Einzelwörter wiedergegeben worden.

Vollends entscheidend ist die beim Arm. ziemlich häufig hervortretende gräcisierende Construction, namentlich die Beibehaltung griechischer Infinitivconstruction, teilweise gar unter Verstößen wider die armenische Syntax und Hervorrufung auffallender Sprachhärten. Diese Gräcismen wären unmöglich so häufig beibehalten und unversehrt bewahrt geblieben, wenn sie vorerst durch das Syrische hätten vermittelt werden müssen.

Dazu kommt außerdem noch, daß, wenn Arm. aus Syr. geschöpft hätte, doch zweifellos die im Urtexte vorhandenen syrischen Eigennamen oder Appellativa in ihrer wahren syrischen Form und nicht in der gräcisierten übernommen worden wären. Nun zeigt aber Arm. die gräcisierte bzw. hellenistische Form; so z. B. Sabinas als Beinamen des Seleukidenkönigs Alexandros $=$ gräcisiert $\Sigma \alpha \beta \iota v \alpha \varsigma$, Siripides als Beinamen des Seleukiden Demetrius II. = hellenist.-syr. $\Sigma \eta \varrho \iota \pi i \delta \eta s$.

Aus vorstehender Betrachtung, die sich in erster Linie auf das I. Buch der eusebischen Chronik bezog, hat sich herausgestellt, daß für diesen Teil des eusebischen Werkes alles entschieden auf eine griechische Quellenvorlage hindeutet.

Es erübrigt nur noch, der Vollständigkeit halber eine Anzahl von Floskeln aus diesem Teil hervorzuheben, die allenfalls in ihrer überlieferten Gestalt auf syrischer Vermittlung beruhen mögen. In dem Eusebius, Chron. arm. 
aus Josephos in der Ebräer-Chronographie angeführten Menander-Fragmente wird genannt ein Phönikerkönig Ithôbalos, König der Astartäer,

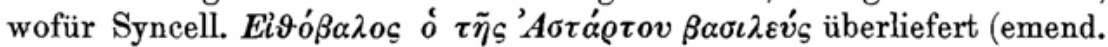

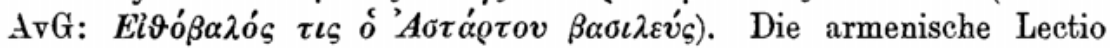
ist gut überliefert, kann unmöglich auf Verlesung der griechischen Originalstelle beruhen, wohl aber auf Verwechslung eines syrischen Status constr. singularis mit Pluralis. In der Römerchronographie kommt die gut verbürgte, aber sinnlose Lectio b Llibtuy i ninea von sNinos\& vor, wo i-y-enea svon Enias» das Richtige wäre; der Fehler erklärt sich leicht unter Annahme, daß Arm. in einer syrischen Vorlage ein richtiges إس position men zu men Nenya od. auch men Ninya verlesen hat. So dürfte denn auch das rätselhafte Demetrios Ola (so wohl zu lesen statt nqug oloç resp. olaç), das außer in dem Seleukidenverzeichnis von T. I noch in der Series regum des Zwischenteils wiederkehrt, am einfachsten durch syrische Vermittelung eines griechischen Demetrios $\alpha v^{3} \vartheta \iota \varsigma$ begreiflich werden ${ }^{1}$.

Als Seitenstück hierzu ließe sich das armenische Epitheton des Königs Ptolemaeos Keraunos anführen, das Arm. regelmäßig durch bark-uthiun Deriv. v. bark ausdrückt; letzteres bedeutet aber nach eigentlichem Sinne sZorn\&, "Groll «, und kommt höchstens in figürlicher Bibelsprache als Semitismus für Blitz in Anwendung. Daß es gleichwohl an dieser Stelle vom Arm. zum Ausdruck von xeqavvós gebraucht wird, da doch sonstige bezeichnendere Termini in Armenisch näher gelegen hätten, wäre geradezu unfaßlich, wenn nicht Arm. aus einer syrischen Vorlage geschöpft hätte, in welcher jenes griech. Epitheton durch syr. barqå sfulgur` ausgedrückt war.

Noch wären vereinzelte Wörter oder Formen zu erwähnen, die in

1) Ola bzw. olotz, olą̧ sind verschiedenartig zu erklären versucht worden. Aucher wollte es mit $\delta \lambda \omega \varsigma$ oder gar ovं $\lambda o \varsigma$ identificieren, während Gutschmid es aus $\alpha \lambda \lambda \alpha$ (scil. - $\left.\tilde{\varepsilon}^{\prime} \tau \eta \delta^{\prime}\right)$ herleiten möchte. Aber auch, falls letztere Conjectur das Richtige träfe, so wäre doch syrische Vermittelung für die Vocalisierung (Vocaltrübung) gewiß anzunehmen. Statt dieser Conjecturen möchte ich in ola eher die Transkription eines syrischen Ausdrucks für den Begrift "prior, anterior" ( ${ }^{2}$ der Vorige $=$ Vorhingenannte«), was für Demetrius II. passen würde, suchen, und das vom Armenier offenbar nicht verstandene Wort zu sem. $\sqrt{\text { avl }}$ stellen, vgl. arab. آل âl «rediit* آول avval *früherer, voriger*. Ob ähnlicherweise auch etwa das rätselhafte Epitheton Tratrẹs, das in dem aus Ktesias überliefertem Fragmente der Serie der Assyrerkönige, vorkommt, durch falsche Transkription vom Syr. aus $\vartheta v \gamma \alpha \tau \varrho o ́ \varsigma$ hervorgegangen sei, auf welches es A. zurückführen will, mag dahingestellt bleiben. 
ihrer auffallenden, weder aus dem Armenischen noch aus dem Griechischen zu deutenden Lautgestalt auf syrische Vermittlung hinzuweisen scheinen: Romelos (st. Romilos), Dẹodoros (st. Demagoras, durch Verwechslung von Mim mit Vav), Lakoriges (st. Lykurgos), Inotos (f. Oinôtros), Krisos (f. Kroisos), Koloe als Schlachtfeld, das doch wohl das thrakische Choloê sein soll, durch Verwechslung bzw. Verlesung des Lautwerts vom syr. Kåf.

-Alles in allem sind dies indessen nur vereinzelte, versprengte Floskeln, die überdies nicht einmal durchgehends sicher und in ihrer Erklärung einwandfrei sind. Im günstigsten Falle kann es sich nur um das Residuum einer nachträglichen Collation mit einem syrischen Original bzw. einer aus syrischem Original geflossenen armenischen Secundär-Version handeln, ein Residuum, das übrigens kaum zur Geltung kommt und so verschwindend unbedeutend ist, daß es an der Tatsache wesentlich nichts ändert, daß der erste Teil des eusebischen Werkes, die eigentliche Chronographie, direct aus griechischem Original übersetzt ist.

\section{Die Syrische Version als Secundärquelle für die Text-Gestaltung des Armeniers.}

Anders gestaltet sich die Sachlage für das zweite Buch, den sog. Kanon. Schon Zohrab und ganz besonders nachdrücklich Petermann' haben auf den Sondercharakter dieses Teiles hingewiesen, auf die Ungleichmäßigkeit der Sprache, die weniger sorgfältige Interpretation, kurzum die anffallenden Abweichungen und Abstände vom Teile I ${ }^{2}$. Hier, im Kanon, ist directer syrischer Einfluß für jeden unbefangenen Beurteiler deutlich erkennbar, und nur einseitige Voreingenommenheit und Verkennung der augenscheinlichsten Tatsachen war es, was Alfred v. Gutschmid veranlaßte, denselben in Abrede zu stellen ${ }^{3}$. Unter ausdrücklicher Verweisung und Bezugnahme auf die diesbezüglichen vortrefflichen Ausführungen Petermanns ${ }^{4}$ beschränken wir uns im Folgenden anf die Feststellung der wesentlichsten einschlägigen Spracherscheinungen, die ein syrisches Original voraussetzen.

1) Ed. Schoe. II p. LiII.

2) Zohrab-Mai Ed. Intr. p. XlX äußert den Zweifel, ob nicht der armenische Kanon des Euseb einen andern Autor zum Interpreten gehabt habe als das I. Buch. Der Zweifel stützt sich 1) auf die größere Fehlerhaftigkeit der Interpretation, 2) auf die minder vortreffliche haikanische Diction dieses II. Teils.

3) AvG, Kl. Schr. I 418.

4) Ed. Schoe. II p. LIII-LV. 
Fälle von mangelhaftem bzw. von dem griechischen Original abweichendem Vocalismus, die sich nur durch Vermittelung des Syrischen erklären lassen:

Phalminios (st. Phlaminios) a. 2271;

Kliad (st. Kal $<1>i a d)$ a. 1536;

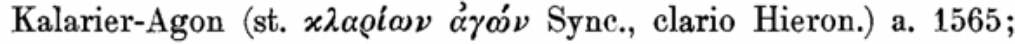

Hyrakania (st. Hyrkania) a. 2033;

Patara ( $\left.\varepsilon i_{\varsigma} \Pi \Pi_{\alpha}^{\prime} \tau \rho \alpha \varsigma\right)$ a. 2001;

Pilinios (st. Plinios) a. 2123;

Isthimin (st. Isthmia) a. 667;

Trianos (st. Traianos) a. 2123;

Bitalios (st. Bitelios) a. 2084;

i-Srnen (st. i-Srenen) »von der Sirenes a. 847;

Platiens (st. Platees) a. 1538, u. a. dgl.

Hierher gehört wohl auch Thermupolis a. 1537 u. a. 1825, bzw. Thermopilis, was wohl ursprünglichere Lesung ist, st. Thermopiles.

Einiges von P. hierher Gerechnete mag sich ja wohl auch auf anderem Wege erklären lassen. Indessen, mag auch als zutreffend oder mindestens annehmbar und wahrscheinlich zugegeben werden, was Gutschmid ${ }^{1}$ hierüber behauptet, daß nämlich sich manches von P. auf Rechnung des Syrischen Gesetzte genügend aus der Geschichte des armenischen Vocalismus erklärt ${ }^{2}$; mag auch eine ganze Reihe weiterer Abweichungen, nach Gutschmids Erachten, durch eine Umschreibung ins Syrische nichts von ihrer Auffälligkeit verlieren (so z. B. Psaneauthes für Psamuthes) oder gar nur auf geringfügigen Schreibfehlern beruhen; mag auch Verschiedenes als Ausfluß armenischer Laut-

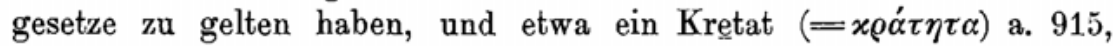
Menaleos (= Menelaos) a. 823 durch bloße Metathesis, ein Bakchalidẹs a. 1550 und Eudomos (für Eudemeos) a. 1125 durch Vocalausgleichung modificiert, ein Krisos aus der daneben erscheinenden Dublette Kriusos oder Krysos a. 1467 abgeschliffen sein - wiewohl es im allgemeinen weit kritischer und sprachgeschichtlich richtiger sein dürfte, jene Entstellung der. Urformen aus dem combinierten Grunde der Wirkung armenischer Lautgesetze und des Durchgangs durch das Syrische

1) Kl. Schr. 1, 437-440.

2) So in der Tat Formen wie Kandoles für Kandauless, Orthopaulis für Orthopolis, Polos für Paulos u. dgl., die, soweit nicht gar Copistencorruptol (Ersatz von älterem uц durch o oder $n$ ) vorliegt, leicht als willkürliche oder inconsequente Schreibungen des armenischen Interpreten gefaßt werden können. 
Einleitung. VIII. Die Syr. Vers. als Secundärquelle f. d. Textgestaltung d. Arm. XLV

hervorgehen zu lassen ${ }^{1}$, so z. B. bei Fällen wie Asklipides gegen gr. Asklepiades a. 2228, Didas gegen gr. Diadas a. 481, Tyrdelianos gegen gr. Tertullianos a. $2051-$, so bleibt dennoch trotz alledem soviel des Auffälligen, nach dem Syrischen Hinweisenden, daß eine wissenschaftliche Forschung sich unmöglich dem wahren Sachverhalte gegenüber verschließen kann.

Wie z. B. vermag Gutschmid eine Form wie Pronikos, die Vers. Arm. a. 1532 als Entsprechung von Phrynichos des Originales steht, anders zu erklären als mittels des Syrischen? Pronikos ist Verlesung aus syr. Phrunikos, indem dem doppeldeutigen Zeichen 9 hier zu Unrecht der nichtaspirierte Lautwert $\mathrm{p}$ und ebenso dem Kå statt des aspir. Lautwertes $\chi$ derjenige der Tenuis $\mathrm{k}$ beigelegt wird.

Auf analoger Verlesung des Armeniers, der die an sich doppeldeutigen Zeichen der syrischen Vorlage: $ص(b-v)$, (ch- ha),,$(k-c h)$, ـ ( $p-f)$ unrichtig faßt, beruht eine ganze Reihe weiterer Abweichungen vom griechischen Original:

So a. 1649 Nechtanebos (st. Nektanebos);

a. 1973 Ochtavios und Hochtavios gegenüber Oktavios in T. l;

a. 2102 Hochtember ${ }^{2}$ ('Ox

a. 2102 Sechtember ${ }^{3}$ ( $\left.\Sigma \varepsilon \pi \tau \varepsilon \dot{\varepsilon} \mu \beta \iota \iota \varsigma\right)$.

Umgekehrt erscheint die Aspirata zur entsprechenden Tenuis verlesen:

in Charkedon, bzw. Chalkedon aus Charkedon (statt Karchedon $K \alpha \varrho \chi \eta \delta \omega^{\prime}$ ) a. $978,1005,1166$, chalikd-aci d. i.: „Chalkidier (statt Karchedonier) a. 1624. Daß wir in diesem Falle wirklich Verlesung aus Syr. bzw. Beeinflussung durch eine syrische Vorlage anzunehmen

1) Hierauf, auf die Beeinflussung griechischer Lehnwörter im Armenischen durch das Syrische bzw. die syrische Vermittelung griechischer Lww. für das Armenische, ist allzuwenig Bedacht genommen auch von Brockelmann in seinem trefflichen Aufsatz über die griechischen Fremdwörter im Armenischen (ZDMG 47 p. 1-42), der alle Modificationen, welche solche Fremd- und Lehnwörter erleiden, ausschließlich durch die Wirkung armenischer Lautgesetze zu erklïren versucht, wiewohl in vielen Fällen syrische Beeinflussung der von ihm angeführten ursprünglich griechischen Worter handgreiflich ist.

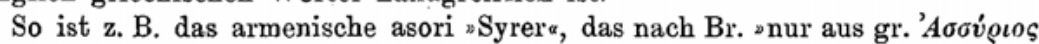
entlehnt sein kann", sicher durch das Syrische übermittelt und ist A. de Lagardes Vergleichung mit אתורי in diesem Sinne ganz richtig.

2) Hochtember oder Hochtembrios erscheint hier bei Fuseb zum ersten Male in der armen, Literatur; vgl. die syrische Entsprechung Octombrios in der alteren Epitome syr. a. 2103. Spätere gelehrte Umbildung ist Hoktember (k st. ch).

3) Sechtember oder Sechtembrios durch Verlesung aus dem Syrischen: م (Koph mit Lautwert $\mathrm{ch})$ anstatt $(\mathrm{Phe}=\mathrm{p}, \mathrm{ph})$. Allerdings auch vulgär armenisch Sektember, das jedoch erst spät nachweisbar ist. 
haben, folgt daraus, daß im ersten Buche der Chronik stets die richtige, dem griechischen Original entsprechende Form Karchedon vorkommt. Durchaus hinfällig erscheint der Einwand Gutschmids, Chalkedon statt Karchedon gehöre zu den gewöhnlichsten Verwechslungen griechischer Handschriften, zumal da die erhaltenen griechischen Correlatstellen die richtige Lesung zeigen. Vielmehr fällt ins Gegengewicht der Umstand, daß wirklich im Syrischen unter Verwechslung derselben Termini die Form صنبم qarqedön und qarqidön für $\boldsymbol{X} \alpha \lambda$ $x \eta \delta \boldsymbol{v}^{\nu}$ neben dem regelmäßigen qalqëdōn belegt ist.

Noch entschiedener weist auf syrische Vorlage zurück die Form skima als Entsprechung für gr. $\sigma \chi \tilde{\eta} \mu \alpha$ in der a. 718 belegten Verbindung wn b ulhufiu (so zu lesen st. hsch. wn lulfibur; vgl. Text). Arm. skima ist als Wiedergabe von gr. $\sigma \chi \tilde{\eta} \mu \alpha$ rein unmöglich: $\sigma \chi \tilde{\eta} \mu \alpha$ hätte nur durch skhima (sk ima) wefufiu oder allenfalls noch schima $u$ lutufu transscribiert werden können; die Tenuis-Form skima beweist peremptorisch, daß der Ausdruck von Arm. nicht aus griechischem, sondern aus syrischem Original genommen worden, indem das Koph dieses Originals statt durch die Aspirata unrichtig durch die Tenuis transcribiert ward.

Auf syrische Vermittelung deuten ferner hin: a. 618 Perinthos

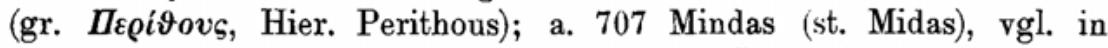
T. I. Nomintor (st. Nomitor); a. 475 Nipha (st. Nymphe); a. 1963 Alipia $^{1}$ (st. Olimpia); sodann a. 812 Melestheus (st. Menestheus); a. 1242 Phalsar (st. Phanassar). Falsche Transcription dürfte wahrscheinlich vorliegen in dem Falle a. 802 Adrametos (st. Adrastos), ibid. Amentes (st. Amenemes). Ferner bei a. 1615 Syraks (st. Syrakus); a. 1353 Ardẹs resp. Ardeos (st. Ardis, resp. Ardisos); a. 813 Didêlos (st. Dẹdalos, $\Delta \alpha i \delta \alpha \lambda o c)$; a. 1989 Sextos (st. Sextilios); 1939 Lipios (st. Lepidos) ${ }^{2}$; vgl. auch Servios (st. Servilios) Ser. Reg. (Ed. p. 150, 2).

Sicherlich aus dem Syrischen verlesen und nur durch syrisches Mittel erklärbar sind Entstellungen wie:

Libyer für Lesbier a. 1345; Egipter für Egineten a. 1530; Egipt $\langle o s\rangle$ für Ego-pot〈amos〉a. 1552; Sidon für Sinope a. 1386; Samarier für Samier a. 1997; Kiprer für Kimbrer a. 1915.

1) Vgl. Ulyp, Dubl. Ulip, Nom.pr. eines altarmenischen Schriftstellers, ebenfalls von dem Syrischen übermittelt $=$ gr. Olympios.

2) Hierber dürfte auch gehören die synkopierte Form Vespianos, das armenische Correlat von Vespasianos, das wohl zunächst aus dem Syrischen transcribiert ward in der Form Vespsianos, die dann zu Vespianos vereinfacht wurde. Dahingestellt mag es freilich bleiben, ob diese Form nicht schon voreusebianisch ist. Vgl. übrigens auch das lat. Nom. pr. Vipsanius (Agrippa). 
Finleitung. VIII. Die Syr. Vers. als Secundärquelle f.d.Textgestaltung d. Arm. XLVII

Besonders merkwürdig ist der Fall, wo durch Verlesung eines syrischen Ee zu Lomad $\checkmark-\mathbb{v}$ geographische Namen durch ein ilElement erweitert werden. So wird bei Arm. ursprüngliches Amyos zu Amilos a. 1376, Dyrrhachion zu Dyr $\langle\mathbf{r}\rangle$ akilon a. 1391.

Als drastischen Fall von Apokopierung oder Verstümmelung eines Nom. propr. führt P. an a. 857 die Stelle svon Chereus $\boldsymbol{k} \boldsymbol{p}^{\boldsymbol{t}} \boldsymbol{p} \boldsymbol{t}$ als

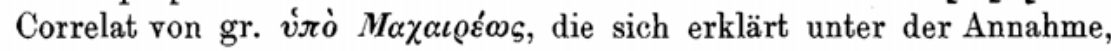
$\mathrm{da}$ der Armenier gelesen habe mit Übersehung des einen $x$.

Umgekehrt erscheinen Fälle von Worterweiterungen durch falsche Zuziehung von Präfixen oder Suffixen: a. 2121 Menesia, was nach P.s treffender Bemerkung (Ed. Schoe. II p. LV) nicht aus gr. $\mu \dot{v} \nu$ 'A $\sigma_{i \alpha \varsigma}$ entstanden sein kann, da es alsdann menasia lauten würde, sondern

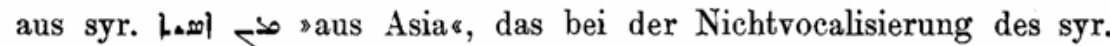
Textes sowohl Menasia als Menesia gelesen werden konnte 1. - Analog erklären sich wohl auch: a. 1469 Adelphier für Delphier, Area T. I (Röm. Chronogr.) für Rhea, und a. 2010 das auffällige 'Arier', wo T.

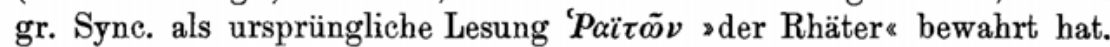

Klassische Beispiele ferner für die Verwechslung einander ähnlicher Buchstaben der syrischen Vorlage, wodurch im Arm. neue Namen herauskommen, sind:
a. 1939 Lipios st. Lepidos,
a. 864 Agenor st. Antenor,
a. 2053 Phlavos st. Phlakos,
a. 1874 Pompeos st. Publios.

Namentlich letzteres Beispiel ist ein frappanter Beleg für den Durchgang durchs Syrische, da in diesem Falle die Form Publios schlechthin unmöglich aus dem Griechischen erklärbar wird, anderseits Corruptel in Arm. ganz ausgeschlossen ist.

Nicht minder frappant offenbart sich die Benutzung einer syrischen Vorlage in folgendem weiteren Falle: a. 2102 lesen wir nach der arm. Überlieferung, die übrigens durch Wiederkehr derselben Lesart bei Sam. An. Chron. gut verbürgt ist, die rätselhafte Namensform Parthenikos als angebliche von Domitian dem MonatOctober beigelegte Neubenennung. Dieser Name ist jedoch ein geschichtliches Unding, ganz abgesehen davon, daß die Form sprachlich verdächtig ist, da für „Parthicus» Arm. sicher das gemeinarmenische Äquivalent Parthev, resp. adj. Parthevakan ge-

1) Saint-Martins Conjectur IJ (ru/ruy Mysia st. Menesia scheitert an der übereinstimmenden Überlieferung von Sync., Hier. Can. und Epit. Syr., die sämtlich die Lesung Asia bieten (Journal des Savans 1820 p. 105). 
schrieben hätte. Augenscheinlich ist Parthenikos verlesen aus einem Brṭkks = Bretañikos der syrischen Vorlage.

Mag auch einzelnes unter diesen Abweichungen und Absonderlichkeiten sich einfach deuten lassen, etwa durch bloße Verschreibung oder Verhörung - unsere Version scheint nämlich, wenigstens teilweise (zumal nach Cod. E), dem Schreiber in die Feder dictiert worden zu sein, woraus sich freilich manche falsche Wiedergabe von Eigennamen und dgl. erklären mag -, so ist diese Annahme doch völlig unzureichend zur Erklärung der großen Masse der auffälligeren Abweichungen, und versagt bei syntaktischen Abweichungen schlechthin.

Im übrigen sei auf das von P. (Ed. Schoe., II. Prol. LIV sqq.) beigebrachte Material verwiesen, aus dem sich vollends ergibt, daß nicht nur einzelne Worte und Ausdrücke, sondern auch ganze Phrasen und Satzteile in ihrer Abweichung vom griechischen Original bestimmt auf syrisches Mittel hinweisen. Von den daselbst angeführten Belegen sind namentlich zwei von entscheidender Beweiskraft:

1. a. 2261: [25 wurde genannte. Corruptel von $\dot{\varepsilon} x \alpha v^{\prime} \vartheta \eta$ zu etwaigem $\dot{\varepsilon} x \lambda \eta^{\prime} \vartheta \eta$ ist hier ganz unwahrscheinlich, die armenische Lesung ist gut überliefert und

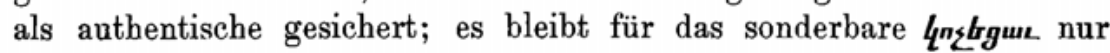
die einzige Erklärungsmöglichkeit, daß Arm. den betr. syrischen Originalausdruck seiner Vorlage falsch gelesen habe; und in der Tat ist, wie Prichtig gesehen hat, ein zu supponierendes der syr. Vorlage $\mathrm{zu}$ i 24 verlesen worden.

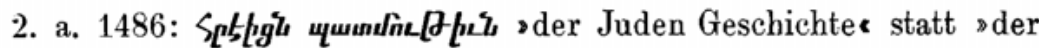
Judith Geschichte . Auch hier ist Corruptel des griechischen Originalausdrucks sowohl wie der gut überlieferten armenischen Lesung schlechthin undenkbar. Es bleibt nur die einzige Möglichkeit: Arm. ist aus Syr. verlesen, wie schon $P$. richtig vermutet hat. Dies ist um so natürlicher und augenscheinlicher, als die entsprechenden syrischen Termini für sJuden" und sJudith" nahezu in der Schrift zusammenfallen. Um so weniger ist an dieser Annahme zu zweifeln, als ebendieselbe verkehrte Lesung auch in der Vorrede des biblischen Judithbuches armenischer Version unterlaufen ist, wo der Fehler anerkanntermaßen auf Verlesung aus syrischer Vorlage beruht.

Zwar hat, in der voreiligen Voraussetzung von der ausschließlich griechischen Provenienz der Vers. armen., A. v. Gutschmid, gegen solche peremptorischen Textzeugnisse hartnäckig ankämpfend, die Benutzung einer syrischen Vorlage durch Arm. in Abrede zu stellen versucht; allein seine diesbezügliche Argumentation, es sei jener (nach 
Einleitung. VIII. Die Syr. Vers. als Secundärquelle f. d. Textgestaltung d. Arm. XIIX

seinem eigenen Zugeständnis) aus syrischer Vorlage herrührende Irrtum, wodurch a. 1486 sJuden mit sJudith * verwechselt wird, schon im armenischen Prolog des Judithbuchs begangen, beweise also Bekanntschaft mit diesem, nicht Benutzung eines syrischen Eusebius, ist nicht stichhaltig und vermag unsere gegenteiligen Folgerungen nicht zu entkräften ${ }^{1}$.

Auf einen syrischen Eusebius führen mit mehr oder minder Wahrscheinlichkeit ferner zurück eine Reihe von fundamental abweichenden Lesungen in Arm.:

a) a. 869 Struulzhqtruligh süS sder Herakliden Tods gegen t. gr.

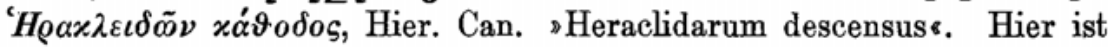
Corruptel der griech. Lectio nicht anzunehmen, ebensowenig solche der armenischen, die gut überliefert ist. Am wahrscheinlichsten ist die Hypothese, der entsprechende syrische Terminus, den Arm. in seiner Vorlage für $x \alpha \dot{\vartheta} \vartheta \delta o \varsigma$ vorgefunden, sei als doppeldeutiger von Arm. im figürlichen Sinne des Hinabsteigens zu den Toten gefaßt, oder auch etwa mit irgendeinem graphisch ähnlichen von der Bedeutung sTode verwechselt worden.

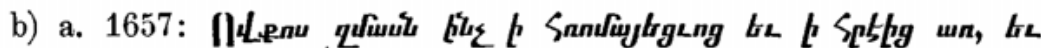

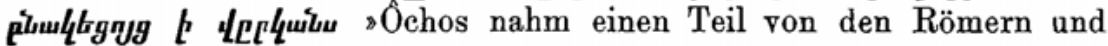
von den Juden und siedelte sie in Vyrkanien anc: gegenüber

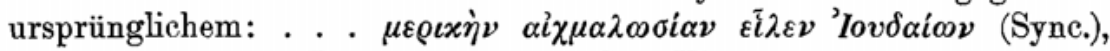
sOchus Apodasmo Judaeorum capta Cod. Hier. Auch hier dürfte vermutlich der Fehler auf Mißverständnis der syrischen Correlatstelle beruhen, ob nun Arm. in Syr. ein Wort der Wurzel hrrm sabschließen,

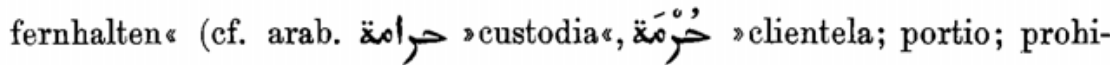
bitum ) oder sonst dergleichen gelesen bzw. zu horom (=arm. »Römers) verlesen haben mag.

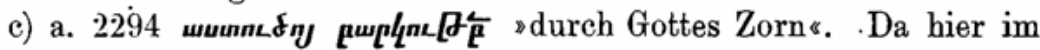

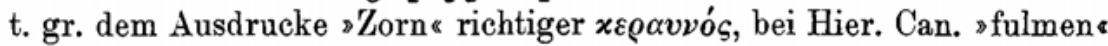
entspricht, dürfen wir hier einen Syrismus annehmen, bzw. daß ein correlates barqå ( Blitz») der syrischen Vorlage bestimmend gewesen sei für die Setzung des phonetisch gleichlautenden arm. purL, deriv.

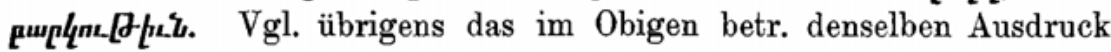
als Eponym des Ptolem. Keraunos Gesagte.

1) Es hieße dies der philologischen Akribie und Wissenschaftlichkeit des Armeniers allzuviel zutrauen und seine gelehrte Arbeitsweise überschätzen, wollte man annehmen, daß derselbe für die fragliche, übrigens nicht geradezu hochwichtige Stelle vorerst noch eigens den betreffenden Bibeltext zu consultieren und zu controllieren für nötig befunden hätte. 
Aber selbst wenn Fällen wie den letztgenannten als problematischen keine eigentlich zwingende Beweiskraft beizumessen sein sollte, so dürfte es doch aus den voraufgegangenen Ausführungen vollauf als erwiesen gelten, daß im armenischen Kanon des Eusebius syrischer Einfluß in starkem Maße vorhanden und daß dieses Element aus einer syrischen Eusebiusvorlage geflossen ist.

Bei dem fundamentalen Abstande der syrischen Syntax von der armenischen brauchte es nicht wunder zu nehmen, wenn Arm. diesbezüglich seine eigenen Wege gegangen wäre und Syrismen, als mit dem armenischen Sprachgeist wenig vereinbar, nicht beibehalten hätte. Dennoch fehlt es nicht an Überbleibseln syrischer Satzbildung und Construction. Schon P. hat auf syntaktische Eigentümlichkeiten, die der syrischen Version entstammen, hingedeutet. Ich erwähne u.a.z.J.Abr. 588:

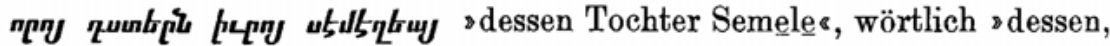
seiner Tochter Semele (Relativsausdruck durch pron. rel. + demonstr.):

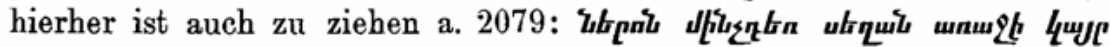
sNeron (Nominativ), während der Tisch ihm vorgesetzt war statt zu er-

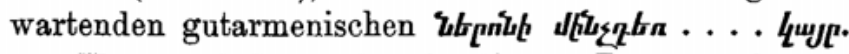

Hervorgehoben seien in diesem Zusammenhange schließlich noch etliche auffällige Constructionen, die auf Verwechslung oder Mißdeutung syrischer Partikeln beruhen:

a. 618: „Des Mädchenraubes Begebenheit bei Aidoneus, dem Molosserkönige«:

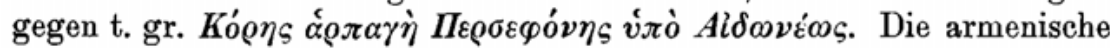
Locativform m. Praep. b sbei, in, zu « erklärt sich aus Syr. Praep. = sb\&, die sowohl zum Ausdruck des Locativus (sbei, in, ans) als auch des Instrumentalis, Causativus (»durch», smittels», saus») dient. Hieran

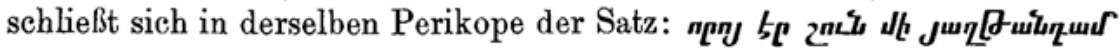

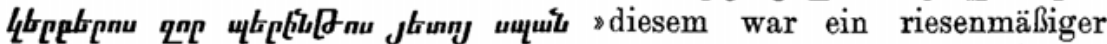
Hund, der Kerberos, den Perinthos nachher tötete«: dagegen $\stackrel{\tau}{\tau} \tau \grave{o} \nu$

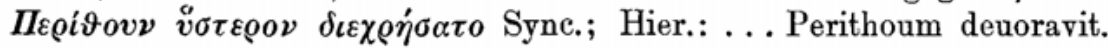

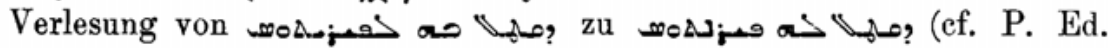
Schoe. LIV).

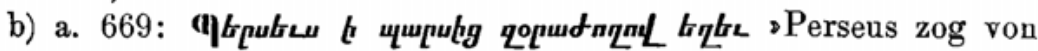

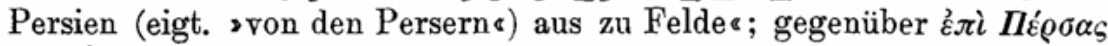

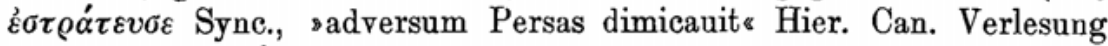
von syr. man ('al) parsôyê zu men al parsôyê. - Vgl. auch a. 813

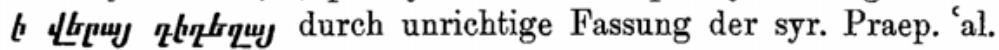

Es würde uns zu weit führen, alle einschlägigen Eigentümlichkeiten des Armeniers erschöpfend behandeln zu wollen, zumal auf 
Einleitung. VIII. Die Syr. Vers. als Secundärquelle f. d. Textgestaltung d. Arm. LI

Grund des Angeführten wir mit hinreichender Sicherheit zu dem Schlusse gelangen, daß in unserem armenischen Eusebiuskanon ein syrisches Original sich widerspiegelt.

Es fragt sich nun, ob die Syrische Version die einzige Quelle ist, woraus unser armenischer Kanon geflossen, oder ob sie mit einer anderen, heterogenen Quelle darin zusammengeflossen sei. Hierauf ist zu erwidern: Die armenische Fassung des Kanons hat keineswegs den Character eines einheitlichen Werkes: in einigen Partien ist syrischer Einfluß unverkennbar, in anderen wiederum kommt eine entschieden griechische Originalvorlage deutlich zum Durchscheine. Insbesondere tiefgreifend sind die sprachlichen und stilistischen Differenzen innerhalb verschiedener Teile der Chronik: bald ist die Diction eine mehr puristische, nationalarmenische, bald wieder verfällt sie in Abhängigkeit und servile Anlehnung an die Quellenvorlagen. So wird meist der Originalname Zeus beibehalten, mitunter indessen auch durch das armenische Aramazd ersetzt (vgl. a. 519, 764, 1850.)

Gr. $\varphi v \sigma \iota x o ́ s$ erscheint vorwiegend in seiner unveränderten Urform recipiert, oder doch nur leicht armenisiert als p'yskian a. 1540, 1560; daneben aber auch a. 1479, a. 1483 dafür die armenische Entsprechung axtarak. Namentlich bezeichnend und bedeutungsvoll ist die Ausdrucks-

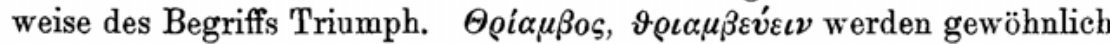

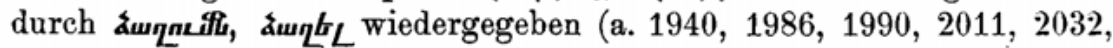

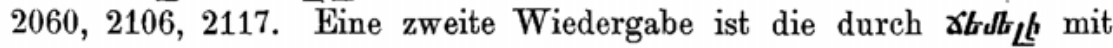

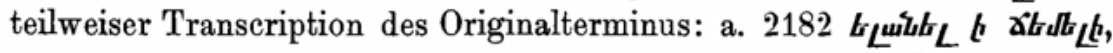

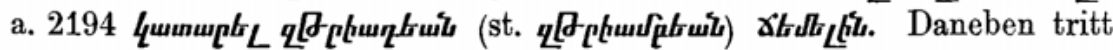
noch als dritter Ausdruck das auch im N. T. Col. 2, 15 vorkommende

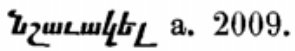

Ganz undenkbar wären dergleichen zum Teil recht wesentliche Unebenheiten und Differenzen innerhalb eines und desselben Werkes, falls dasselbe das aus nur einer einzigen Vorlage herrührende Erzeugnis eines einzigen Übersetzers oder Redactors wäre. Aber auch die Annahme, daß unsere Version von mehreren Übersetzern etwa als Mitgliedern ein und derselben Schule aus einem einheitlichen Original angefertigt sei, wäre unzureichend zur Erklärung der Inconsequenzen in Sprache und Fassung. Die einzig natürliche, mit zwingender Logik sich ergebende Schlußfolgerung ist diese: Die armen. Version des Euseb.-Kanons ist als Fortsetzung des ersten Buches der Chronik ursprünglich und ibrem Grundstocke nach gemeinschaftlich mit jenem ersten Teil direct aus dem griechischen Original geflossen. Sie hat jedoch nachträglich, aus naheliegenden Gründen, sei es, daß jene erste dem Griechischen entstammende 
Übertragung eine unvollständige, sei es daß sie eine defecte bzw. ungenaue und unbefriedigende gewesen, eine Überarbeitung erfahren auf Grund eines syrischen Originals, so zwar daß die mangelhaften Partien und Defecte durch Collationierung aus jener syrischen Vorlage bzw. deren armenischer Version ergänzt und interpoliert wurden. Dadurch erhielt die ursprüngliche griechisch-armenische Version des Kanons ein bedeutendes syrisch-armenisches Element zum Einschlag, derart daß in gewissem beschränkten Sinne für diesen II. Teil wohl von Contamination zweier an sich verschiedener Versionen gesprochen werden $\operatorname{darf}^{1}$, während der I. Teil der Chronik einen im ganzen einheitlichen Charakter trägt und nur ganz vereinzelte Floskeln aufweist, die nachträglich aus syrischer bzw. syrisch-armenischer Vorlage hineinkorrigiert bzw. interpoliert worden sein mögen.

Ins Syrische übersetzt wurde die Eusebische Chronik zum ersten Male von Simeon dem Garmakäer (Šem iun Garme ${ }^{\circledR}$ ojô) um das Jahr $600 \mathrm{n}$. Chr. Nahezu um dieselbe Zeit ist nach unseren vorhin gegebenen Ausfübrungen auch die ursprüngliche armenische Version derselben Chronik aus dem griechischen Archetypus hergestellt worden. Darum wäre es an sich keineswegs undenkbar, daß schon der Urheber der ursprünglichen armenischen Übersetzung, d. h. der griechisch-armenischen Übersetzung, jene oben besprochene Collation seines Textes mit dem des syrischen Originals vorgenommen habe. Jedenfalls dürfte jene Überarbeitung und Contaminierung des ursprünglichen, aus dem Griechischen hervorgegangenen Textes mit der syrischen Version bzw. deren armenischen Übertragung sehr frühzeitig, und zwar mindestens vor dem Ende des 8. Jhds. erfolgt sein. Dies geht hervor 1) aus der continuierlich gleichmäßigen Textüberlieferung der armenischen Chronik, die sich in den Excerpten und Epitomen der armenischen Historiker ausdrückt, während doch, wenn erst später, etwa im 9. Jhd., die neue Textrecension erfolgt wäre, dieses durch Differenzen zwischen den von den Schriftstellern jener Zeit angeführten Excerpten und denen der früheren Jahrhunderte zum Ausdruck kommen müßte; 2) aus der Tatsache, daß die syrische Version, welche den Einschlag zu jener Recension des eusebischen Werkes, speciell des Kanons, abgegeben hat, wirklich keine andere als jene erste syrische Übersetzung, d. i. die des Simon Garmeqojô, ist, von der im J. 636 ein Auszug gefertigt ward, der, obgleich

1) Eine Reihe analoger Doppelredactionen von altarmenischen Versionen auf Grund von zwei Vorlagen, einer griechischen und einer syrischen, stützen und bestätigen diese Schlußfolgerung. Man denke nur an die Geschichte der armenischen Bibelversion. 
Einleitung. VIII. Die Syr. Vers. als Secundärquelle f. d. Textgestaltung d. Arm. LIII

nur Auszug, doch in wunderbarer Weise mit unserem Kanon zusammenstimmt. Wäre dagegen die fragliche Recension des Armeniers an der Hand syrischer Vorlage erst später, beispielsweise im 8.-9. Jhd. erfolgt, so wäre gewiß nicht jene alte syrische Version, sondern vielmehr die gleichzeitige, ausgangs des 8. Jahrhunderts von Dionysios v. Tellmahrê verfaßte, zugrunde gelegt worden.

Der erwähnte, bisher übersehene Umstand der engen verwandtschaftlichen Beziehung der syrischen Epitome des Euseb-Kanons v. J. 636 mit unserem armenischen Kanon ist von nicht unerheblicher Bedeutung für die Textkritik unserer Version und die Reconstruction defecter oder schwieriger Textstellen. Die Collation der Epitome stellt im allgemeinen in dieser Beziehung ein sichereres Kriterium dar, als die übrigen in Betracht kommenden Parallelrecensionen: Can. Hieron., Sync. Can., Fragm. gr.

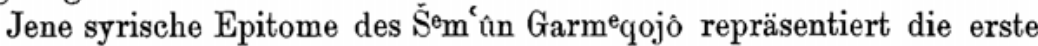
oder Originalausgabe der griechischen Euseb-Chronik, womit auch stimmt, daß im ganzen und großen die armenische Version denselben Charakter jener I. Ausgabe trägt, so namentlich bezüglich der Papstlisten, die beim Armenier gegenüber Vers. Hieron. die ursprüngliche Eusebianische Fassung bewahrt haben. Aber gleichwohl ist unsere armenische Fassung nicht frei geblieben von Elementen, die ebenso unverkennbar der II. revidierten Ausgabe des Originalwerkes angehören. Hierher gehört vor allem die nachträglich zwischen Buch I und II interpolierte Zwischenpartie der sog. Series regum, die, wie Schoe. Weltchron. überzeugend dargetan hat, sich als ein Auszug aus den Königslisten der zweiten, revidierten Originalausgabe darstellt, dazu bestimmt als Correctiv-Beilage zu der alten, die ursprüngliche oder I. Ausgabe vertretenden Version zu dienen, sin demjenigen Hauptbestandteil, in welchem die neue Ausgabe am stärksten von der älteren abwich «. Hierher sind sodann wohl auch zu rechnen die auf Grund dieser neueingeführten verbesserten sKönigs-Reihen " mehrfach an den Königslisten der Kanones offensichtlich gemachten Correcturen, wodurch letztere mit jenen in Übereinstimmung gebracht werden sollten, ein Punkt, auf den im App. critic. noch eingehender zurückzukommen sein wird.

So ist denn Lightfoots (Chr. Biogr. 2 p. 322. 330) geäußerte Ansicht, $\mathrm{da} ß$ in der armenischen Chronik-Version eine Mischung der früheren und der späteren Ausgabe des Euseb-Werkes zu erkennen sei, wesentlich richtig; nur darf nicht etwa an eine tiefgreifende Umgestaltung des ursprünglichen, die I. Editio vertretenden. arm. Textes aus der IIten gedacht werden, sondern lediglich an eine oberflächliche Conta- 
mination bzw. Interpolation, wodurch die ursprüngliche Textfassung als solche nicht wesentlich gestört wurde.

$\mathrm{Da}$ ferner die fraglichen sSeries Regum» unserer Codices nach vereinzelten Floskeln (wie z.B. Demetrios Olatz) deutlich ihre syrische Provenienz verraten, so müssen sie aus syrischer Quelle geflossen sein. Als solche kann schlechthin nur die oben erwähnte syr. Chronik des Dionys. Telmaharensis in Betracht kommen, eine Epitome der Euseb-Chronik, die im Gegensatz zu der alten syr. Epitome vom J. 600 n. Chr. aus der zweiten Ausgabe der Originalchronik geflossen ist. Hieraus folgt weiter, daß als terminus post quem für diese 2. Revision der ursprgl. armenischen Euseb-Version etwa der Beginn des 9. Jahrhunderts anzusetzen ist.

Möglich, ja wahrscheinlich dürfte diese 2. Revision sich bloß auf den Kanon erstreckt haben. Ist doch mit ziemlicher Sicherheit anzunehmen, daß die Überlieferung der beiden Chronik-Bücher schon frühzeitig eine getrennte wurde, indem von dem wesentlich wissenschaftlichen I. Buche, der eigentl. Chronographie, als praktisches Hand- und Nachschlagebuch das Tabellenwerk sich abspaltete; hieraus würden sich dann auch erklären die bedeutenden Textausfälle und Defekte des KanonBuches, wovon der die Anfangspartie betreffende schier undenkbar wäre, wenn dieses II. Buch mit dem ersten durchgehends zu einem einheitlichen Codex-Bande vereinigt in Umlauf gewesen wäre, da in der Mitte eines Bandes doch schwerlich solche Textausfälle vorkommen dürften. 